\title{
Unusual Magnetic Behavior of Six-Coordinate, Mixed-Ligand Re(II) Complexes: Origin of a Strong Temperature-Independent Paramagnetism ${ }^{\dagger}$
}

\author{
Kim R. Dunbar, ${ }^{*}$, Eric J. Schelter, ${ }^{\ddagger}$ Andrei V. Palii, ${ }^{\S}$ Sergei M. Ostrovsky,,$\perp$ \\ Vadim Yu. Mirovitskii, ${ }^{\S}$ Joshua M. Hudson," Mohammad A. Omary, ${ }^{\prime, \otimes}$ \\ Sophia I. Klokishner, ${ }^{*}, \#$ and Boris S. Tsukerblat*, $\nabla$ \\ Department of Chemistry, Texas A\&M University, College Station, Texas, 77842-3012, \\ Institute of Applied Physics, Academy of Sciences of Moldova, Academy str. 5, Kishinev MD-2028, Moldova, \\ Department of Chemistry, University of North Texas, Denton, Texas 76203, \\ State University of Moldova, Mateevich str. 60, Kishinev MD-2009, Moldova, and \\ Department of Chemistry, Ben-Gurion University of the Negev, P.O. Box 84105, Beer-Sheva 84105, Israel
}

Received: April 17, 2003; In Final Form: September 11, 2003

\begin{abstract}
An explanation is presented for the unusual magnetic behavior of two $\operatorname{Re}(\mathrm{II})$ compounds, $\left[\mathrm{Re}(\operatorname{triphos})\left(\mathrm{CH}_{3^{-}}\right.\right.$ $\left.\mathrm{CN})_{3}\right]\left[\mathrm{BF}_{4}\right]_{2}$ and $\left[\mathrm{Et}_{4} \mathrm{~N}\right]\left[\operatorname{Re}(\right.$ triphos $\left.)(\mathrm{CN})_{3}\right]$, for which magnetic susceptibility data obtained on powder samples $(2-300 \mathrm{~K})$ suggest unusually strong temperature-independent paramagnetism $\left(\chi_{\mathrm{TIP}}=(1.4-1.8) \times 10^{-3} \mathrm{~cm}^{3}\right.$ $\mathrm{mol}^{-1}$ ) and small low-temperature effective magnetic moments. A model is developed based on the jj-coupling scheme appropriate for description of the $5 \mathrm{~d}$ shell of a $\mathrm{Re}(\mathrm{II})$ ion in a crystal field. The model accounts for a cubic crystal field, strong spin-orbit coupling, and a trigonal component of the crystal field produced by the ligand field acting on the $\operatorname{Re}(\mathrm{II})$ ions. The last two interactions act within the truncated basis containing eight lowest, strong cubic crystal field terms and result in the stabilization of two closely spaced Kramers doublets originating mainly from the ${ }^{2} \mathrm{~T}_{2}\left(\mathrm{t}_{2}^{5}\right)$ term. Efficient mixing of these Kramers doublets with those arising from ${ }^{6} \mathrm{~A}_{1}\left(\mathrm{t}_{2}^{3} \mathrm{e}^{2}\right)$ and ${ }^{4} \mathrm{~T}_{1}\left[\mathrm{t}_{2}^{4}\left({ }^{3} \mathrm{~T}_{1}\right) \mathrm{e}\right]$ terms is shown to result in the small low-temperature effective magnetic moments and anomalously strong temperature-independent paramagnetism, in accordance with the observed magnetic behavior. The model perfectly reproduces the $\chi T$ vs $T$ dependences over a wide temperature range $(2-300 \mathrm{~K})$, and the energy pattern obtained with the set of the best fit parameters provides a qualitative explanation for the observed light absorption and diffuse reflectance in a wide spectral area $(200-2600 \mathrm{~nm})$. The theoretical consideration predicts extremely strong anisotropy of the magnetic susceptibility and $g$-factors for both compounds with a $C_{3}$ easy axis of magnetization.
\end{abstract}

\section{Introduction}

Magnetochemisty of the iron group complexes is a welldeveloped field of research with a solid theoretical background ${ }^{1,2}$ and reliably established trends of behavior (for reviews, see refs $1-6)$. Often in metal complexes of the first row $3 \mathrm{~d}$ transition metals, the cubic crystal field and the interelectronic interactions are of the same magnitude and dominate the magnet behavior. The spin-orbit interaction is relatively weak in these cases. Theoretical consideration of these systems is based either on weak or on strong crystal field schemes with allowance for configuration interactions. Systems possessing unquenched orbital angular momenta are especially interesting and are typically represented by examples such as octahedral high-spin complexes of $\mathrm{Co}(\mathrm{II})$ and $\mathrm{Fe}(\mathrm{III})$ (ground-state terms ${ }^{4} \mathrm{~T}_{1}$ and ${ }^{6} \mathrm{~T}_{2}$, respectively) and low-spin Fe(III) complexes (ground-state term ${ }^{2} \mathrm{~T}_{2}$ ). The model conventionally used to treat the magnetic and electron paramagnetic resonance (EPR) data of these

\footnotetext{
$\dagger$ This paper is dedicated to Professor Dan Meyerstein on the occasion of his 65 th birthday.

* Corresponding authors. E-mail for K.R.D: dunbar@mail.chem.tamu.edu; E-mail for B.T.: tsuker@bgumail.bgu.ac.il; E-mail for S.K.: klokishner@ yahoo.com or sophia@usm.md.

$\doteqdot$ Texas A\&M University.

$\S$ Academy of Sciences of Moldova.

${ }^{\perp}$ E-mail: sm_ostrovsky@yahoo.com.

"University of North Texas.

${ }^{\otimes}$ E-mail: omary@unt.edu.

\# State University of Moldova.

$\nabla$ Ben-Gurion University of the Negev.
}

systems takes into consideration the splitting of the ground orbital triplet by the spin-orbit interaction and the lowsymmetry components of the crystal field.

In compounds of the palladium and platinum groups, the cubic crystal field is usually assumed to be strong, ${ }^{6}$ and the interelectronic interaction is essentially weakened because of large extension of the $4 \mathrm{~d}$ and $5 \mathrm{~d}$ electronic shells, while at the same time spin-orbit coupling is much stronger than that in the iron group compounds. This situation leads to the breakdown of the Russell-Saunders scheme (in both weak and strong crystal fields) and the appropriate coupling can be adequately described by the $\mathrm{jj}$ (intermediate)-coupling scheme, attributed to Van Vleck. ${ }^{7}$ Using the intermediate coupling scheme, Liehr ${ }^{8}$ considered a one-electron heavy metal ion system with an axially distorted octahedral environment and Moffit et al. ${ }^{9}$ analyzed absorption spectra of hexafluorides of $5 \mathrm{~d}^{n}(n=2,3$, and 4) ions. Although the general theoretical routes to treat the magnetic characteristics of $4 \mathrm{~d}$ and $5 \mathrm{~d}$ ions have been developed, studies of these systems are relatively scarce (see, for example, the survey in ref 6 and references therein) and do not provide a systematic and comprehensive knowledge of the regularities that are intrinsic to these systems. At the same time, because of the interplay between strong spin-orbit coupling and low-symmetry crystal field, these systems are expected to exhibit interesting magnetic properties and, in particular, strong magnetic anisotropy. This important issue is closely related to the phenomenon of "single molecular magnetism" that has received extensive 


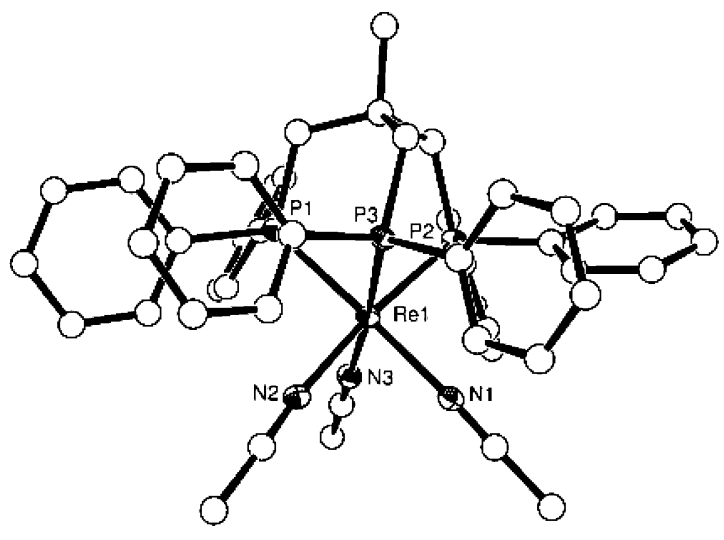

Compound 1

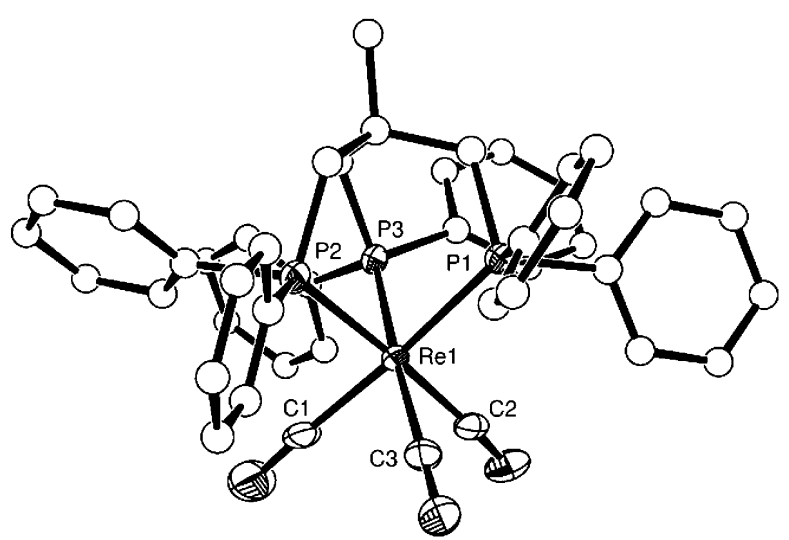

Figure 1. Molecular structures of compounds 1 and 2. Thermal ellipsoids are shown at the $50 \%$ probability level. Carbon atoms are represented as spheres of arbitrary radius unless otherwise denoted.

attention in recent years because of its potential in molecular electronics and quantum computing. ${ }^{10,11}$

The present paper is focused on the study of the manifestation of strong spin-orbit coupling and an axial crystal field in the magnetic properties of heavy metal ions with an outer $5 \mathrm{~d}$ shell. We have investigated the two $\mathrm{Re}(\mathrm{II})$ compounds, $[\mathrm{Re}$ (triphos)$\left.\left(\mathrm{CH}_{3} \mathrm{CN}\right)_{3}\right]\left[\mathrm{BF}_{4}\right]_{2}$ (compound 1) and $\left[\mathrm{Et}_{4} \mathrm{~N}\right]\left[\operatorname{Re}(\right.$ triphos $\left.)(\mathrm{CN})_{3}\right]$ (compound 2), containing the tripodal phosphine ligand $\mathrm{CH}_{3} \mathrm{C}\left(\mathrm{CH}_{2} \mathrm{PPh}_{2}\right)_{3}$ (1,1,1-tris(diphenylphosphino)methyl)ethane, abbreviated triphos). Very little data exist in the literature on the temperature dependence of the magnetic properties of $\operatorname{Re}(\mathrm{II})$ compounds. The available data from our laboratory showed that $\operatorname{Re}(\mathrm{II})$ and $\operatorname{Re}(\mathrm{III})$ complexes exhibit large temperatureindependent paramagnetic susceptibilities. ${ }^{12,13} \mathrm{We}$ attempt to interpret the unusual magnetic properties, measured on powdered samples of these $\operatorname{Re}($ II) complexes exhibiting small $g$-factors and anomalously strong temperature-independent paramagnetism. The proposed extended crystal field model accounts properly for the mixing of the relevant cubic crystal field terms with strong spin-orbit coupling and the trigonal crystal field created by the mixed ligand environment of six-coordinate $\mathrm{Re}(\mathrm{II})$. In a general sense, this model is equivalent to the $\mathrm{jj}$ coupling scheme that is appropriate for the description of a $5 \mathrm{~d}$ shell of $\operatorname{Re}(\mathrm{II})$ in a crystal field. The model provides a perfect fit of the observed $\chi T$ vs $T$ dependences over a wide temperature range $(2-300 \mathrm{~K})$ and predicts extremely strong magnetic anisotropy of the compounds studied. For verification of the theoretical model developed, we also discuss also the optical absorption and diffuse reflectance spectra of the title compounds in a wide spectral range $(200-2600 \mathrm{~nm})$.

\section{Experimental Data}

Detailed synthetic and structural data for compounds $\mathbf{1}$ and $\mathbf{2}$ are described elsewhere. ${ }^{13 a}$ The molecular structures of these compounds with the atomic numbering schemes are shown in Figure 1. The main interatomic distances and angles are listed in Table 1. The magnetic susceptibility data obtained on the powder samples are presented in the form $\chi T$ vs $T$ in Figure 2. The low-temperature limits of $\chi T$ for compounds $\mathbf{1}$ and $\mathbf{2}$ are about 0.291 and $0.205 \mathrm{~cm}^{3} \mathrm{~K} \mathrm{~mol}^{-1}$, respectively. These values correspond to effective magnetic moments of about 1.53 and $1.28 \mu_{\mathrm{B}}$, respectively, which are significantly lower than the pure spin- $1 / 2$ value $\left(1.73 \mu_{\mathrm{B}}\right)$. The fast rate of the linear increase
TABLE 1: Selected Bond Lengths (̊̊) and Angles (deg) for Compounds 1 and $2^{a}$

\begin{tabular}{|c|c|c|c|}
\hline \multicolumn{2}{|c|}{ compound 1} & \multicolumn{2}{|c|}{ compound $\mathbf{2}$} \\
\hline parameter & length/angle & parameter & length/angle \\
\hline $\operatorname{Re}(1)-\mathrm{P}(1)$ & $2.399(10)$ & $\operatorname{Re}(1)-P(1)$ & $2.429(2)$ \\
\hline $\operatorname{Re}(1)-\mathrm{P}(2)$ & $2.384(9)$ & $\operatorname{Re}(1)-\mathrm{P}(2)$ & $2.422(2)$ \\
\hline $\operatorname{Re}(1)-\mathrm{P}(3)$ & $2.391(9)$ & $\operatorname{Re}(1)-P(3)$ & $2.425(2)$ \\
\hline $\operatorname{Re}(1)-\mathrm{N}(1)$ & $2.096(3)$ & $\operatorname{Re}(1)-C(1)$ & $2.038(9)$ \\
\hline $\operatorname{Re}(1)-\mathrm{N}(2)$ & $2.095(3)$ & $\operatorname{Re}(1)-C(2)$ & $2.110(9)$ \\
\hline $\operatorname{Re}(1)-\mathrm{N}(3)$ & $2.094(3)$ & $\operatorname{Re}(1)-C(3)$ & $2.109(8)$ \\
\hline $\mathrm{P}(1)-\operatorname{Re}(1)-\mathrm{P}(2)$ & $88.11(3)$ & $\mathrm{P}(1)-\operatorname{Re}(1)-\mathrm{P}(2)$ & $84.53(7)$ \\
\hline $\mathrm{P}(1)-\operatorname{Re}(1)-\mathrm{P}(3)$ & $85.16(3)$ & $\mathrm{P}(1)-\operatorname{Re}(1)-\mathrm{P}(3)$ & $84.87(7)$ \\
\hline $\mathrm{P}(2)-\operatorname{Re}(1)-\mathrm{P}(3)$ & $88.84(3)$ & $\mathrm{P}(2)-\operatorname{Re}(1)-\mathrm{P}(3)$ & $87.32(7)$ \\
\hline $\mathrm{N}(1)-\operatorname{Re}(1)-\mathrm{N}(2)$ & $82.88(10)$ & $\mathrm{C}(1)-\operatorname{Re}(1)-\mathrm{C}(2)$ & $86.1(3)$ \\
\hline $\mathrm{N}(1)-\operatorname{Re}(1)-\mathrm{N}(3)$ & $84.20(10)$ & $\mathrm{C}(1)-\operatorname{Re}(1)-\mathrm{C}(3)$ & $85.3(3)$ \\
\hline $\mathrm{N}(2)-\operatorname{Re}(1)-\mathrm{N}(3)$ & $85.02(10)$ & $\mathrm{C}(2)-\operatorname{Re}(1)-\mathrm{C}(3)$ & $83.8(3)$ \\
\hline $\mathrm{N}(1)-\operatorname{Re}(1)-\mathrm{P}(2)$ & $91.25(8)$ & $\mathrm{C}(1)-\operatorname{Re}(1)-\mathrm{P}(2)$ & $90.6(3)$ \\
\hline $\mathrm{N}(1)-\operatorname{Re}(1)-\mathrm{P}(3)$ & $99.78(7)$ & $\mathrm{C}(1)-\operatorname{Re}(1)-\mathrm{P}(3)$ & $95.4(3)$ \\
\hline $\mathrm{N}(1)-\operatorname{Re}(1)-\mathrm{P}(1)$ & $175.01(7)$ & $\mathrm{C}(1)-\operatorname{Re}(1)-\mathrm{P}(1)$ & $175.1(3)$ \\
\hline
\end{tabular}

${ }^{a}$ Estimated standard deviations in the last significant digits are given in parentheses.

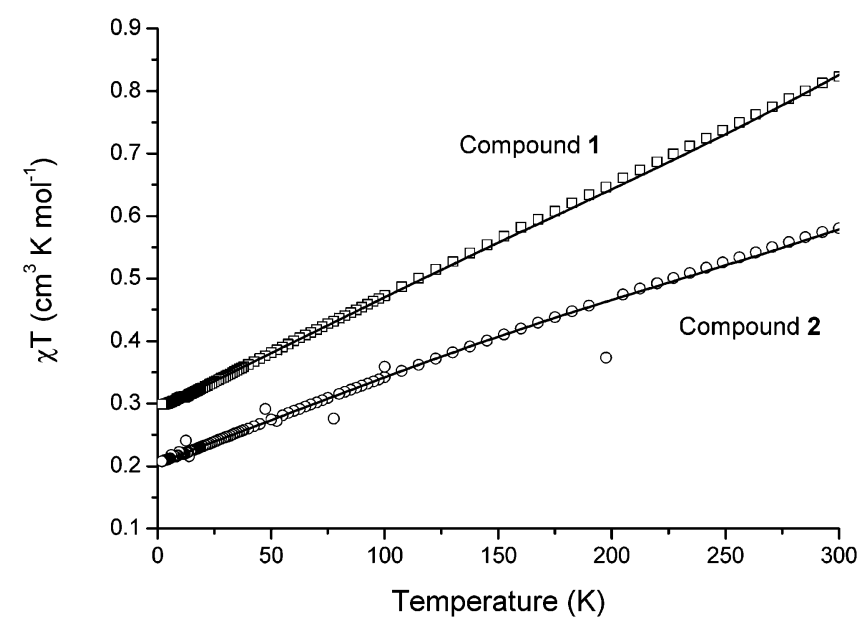

Figure 2. Comparison of the experimental $\chi T$ vs $T$ dependence for $\mathbf{1}$ $(\square)$ and $2(\bigcirc)$ with those calculated with the best-fit parameters $(-)$.

of $\chi T$ vs $T$ over a wide range from 2 to $300 \mathrm{~K}$ demonstrates very strong temperature-independent (Van Vleck type) paramagnetism (TIP). The TIP contributions to $\chi$ are $1.796 \times 10^{-3}$ and $1.363 \times 10^{-3} \mathrm{~cm}^{3} \mathrm{~mol}^{-1}$ for compounds $\mathbf{1}$ and $\mathbf{2}$, respectively. These values are 1 order of magnitude larger than the TIP susceptibilities that are typical for the $3 \mathrm{~d}$ ions. 


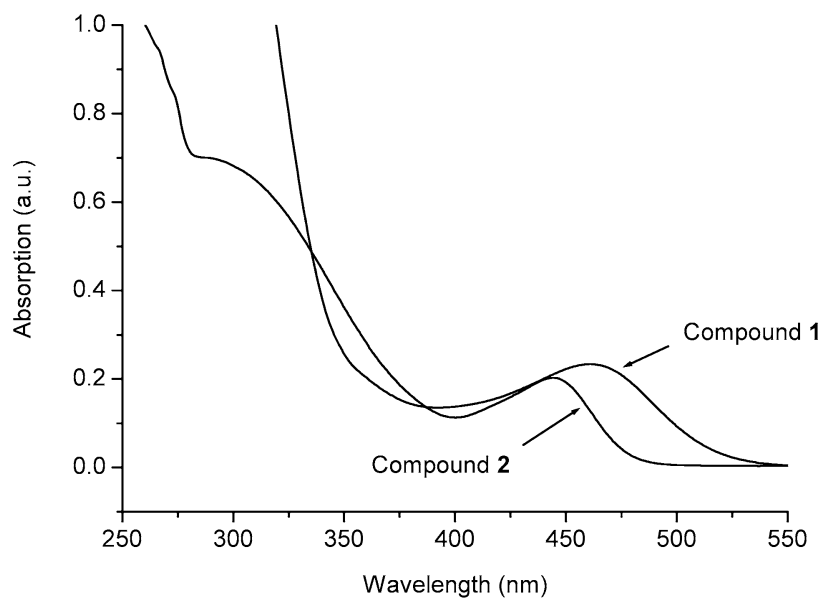

Figure 3. UV-visible absorption spectra for compounds $\mathbf{1}$ and $\mathbf{2}$ in acetonitrile solutions.

UV-visible electronic spectra of $\mathbf{1}$ and $\mathbf{2}$ were performed in acetonitrile solution and recorded with a Shimadzu UVPC-1601 spectrophotometer. The spectroscopy of $\mathbf{1}$ was actually performed on $\left[\mathrm{Re}\right.$ (triphos) $\left.\left(\mathrm{CH}_{3} \mathrm{CN}\right)_{3}\right]\left[\mathrm{PF}_{6}\right]_{2},{ }^{13 \mathrm{a}}$ a compound identical to 1 except for the identity of the counterion. The identity of $\mathbf{1}$ is used interchangeably throughout the paper to indicate $\left[\operatorname{Re}\right.$ (triphos) $\left.\left(\mathrm{CH}_{3} \mathrm{CN}\right)_{3}\right][\mathrm{X}]_{2} \mathrm{X}=\mathrm{BF}_{4}$ or $\mathrm{PF}_{6}$. The spectral measurements in the visible region revealed a broad featureless absorption band between 18500 and $26000 \mathrm{~cm}^{-1}$ (maximum at $\left.21700 \mathrm{~cm}^{-1}, \epsilon=1 \times 10^{3} \mathrm{M}^{-1} \mathrm{~cm}^{-1}\right)$ for compound 1 and between 20000 and $25000 \mathrm{~cm}^{-1}$ (maximum at $22600 \mathrm{~cm}^{-1}, \epsilon$ $=1 \times 10^{3} \mathrm{M}^{-1} \mathrm{~cm}^{-1}$ ) for compound 2 (Figure 3 ). One can assign these bands to spin-allowed $\mathrm{d}-\mathrm{d}$ transitions. A similar band was observed previously in the electronic spectra of the hexahalo anion $\mathrm{IrX}_{6}{ }^{2-}(\mathrm{X}=\mathrm{F}, \mathrm{Cl}, \mathrm{Br})$, wherein the iridium(IV) ion has the same $\mathrm{d}^{5}$ configuration as $\operatorname{Re}(\mathrm{II}){ }^{14}$ The absorption spectra also exhibit intense bands at higher frequencies that can be associated with the metal-ligand charge-transfer transitions.

Diffuse reflectance spectra were measured using a PerkinElmer Lambda 900 double-beam UV/vis/NIR spectrophotometer. The solid-state diffuse reflectance spectra were measured using a 150-mm integrating sphere (by Labsphere) accessory to the Lambda 900 spectrophotometer. This was accomplished by either packing the crystalline solid in a $0.1 \mathrm{~mm}$ Suprasil quartz cuvette (Wilmad) or preparing a thin film of the solid on a filter paper by adding a few drops of a saturated $\mathrm{CH}_{3} \mathrm{CN}$ solution of the relevant compound at the center of the filter paper and letting the solution evaporate to dryness, then crushing a small amount of solid on the filter paper and applying pressure with a spatula to grind it into the paper sufficiently for it to remain fixed. The spectra were measured in a wide range from 2600 to $200 \mathrm{~nm}$ (Figure 4). These spectra are similar for both compounds and demonstrate several distinct peaks in the nearinfrared region $\left(4000-6000 \mathrm{~cm}^{-1}\right)$; in the region 6000-12 500 $\mathrm{cm}^{-1}$ one can observe a region of no transitions and then a relatively strong and structured reflectance band with the major part of the intensity in a wide range, $17000-50000 \mathrm{~cm}^{-1}$.

\section{Crystal Field Consideration}

3.1. The Model. Inspection of the molecular structures (Figure 1) and interatomic distances (Table 1) for $\mathbf{1}$ and $\mathbf{2}$ reveals that both compounds possess a quasi-octahedral ligand environment around the $\operatorname{Re}(\mathrm{II})$ ion that may be regarded approximately as a trigonal system belonging to the $C_{3 v}$-symmetry group. The $C_{3}$ axis passes through triangular faces, one composed of the

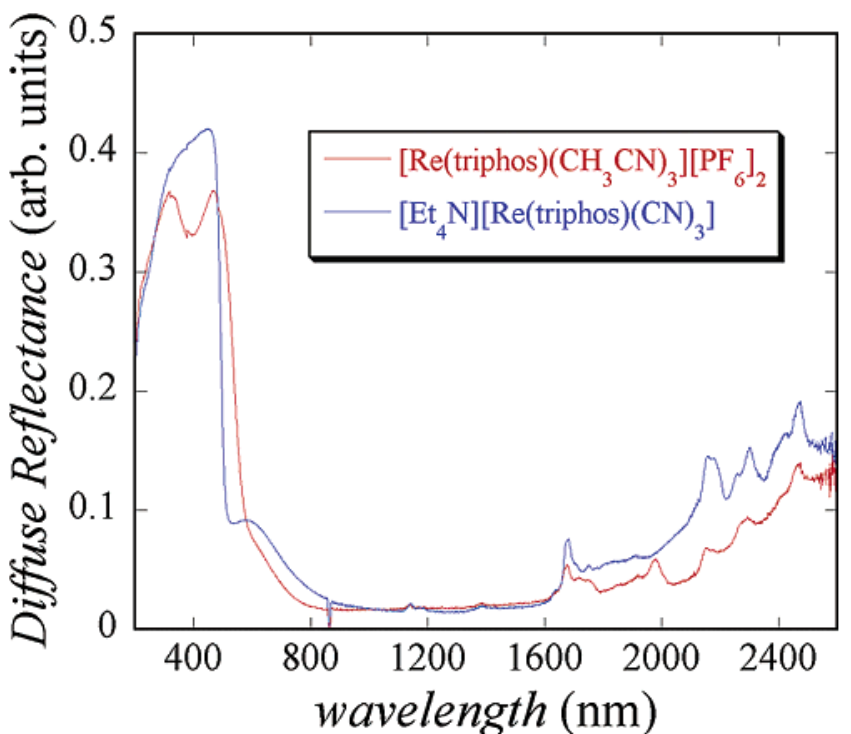

Figure 4. Diffuse reflectance spectra for compounds $\mathbf{1}$ and 2 in the solid state.

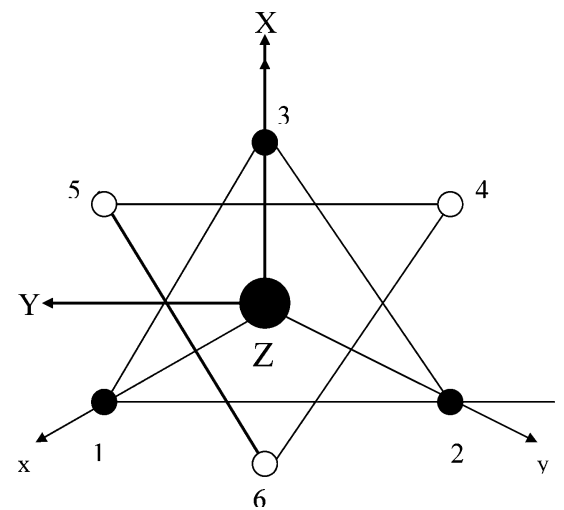

Figure 5. Trigonal coordinate system, $Z|| C_{3}$ axis, $X Y Z$-trigonal coordinates, and $x y z$-tetragonal frame (axes are directed along the $C_{4}$ axes of the octahedron).

phosphorus atoms and the other containing three acetonitrile nitrogen atoms in compound $\mathbf{1}$ and three cyano-carbon atoms in compound $\mathbf{2}$.

The Hamiltonian of the system

$$
\mathbf{H}=\mathbf{V}_{\mathrm{c}}+\mathbf{V}_{\text {trig }}+\mathbf{V}_{\mathrm{ee}}+\mathbf{H}_{\mathrm{SO}}+\mathbf{H}_{\mathrm{Z}}
$$

includes cubic and trigonal crystal fields $\left(\mathbf{V}_{\mathrm{c}}+\mathbf{V}_{\text {trig }}\right)$, interelectronic interactions $\left(\mathbf{V}_{\mathrm{ee}}\right)$, spin-orbit coupling $\left(\mathbf{H}_{\mathrm{SO}}\right)$, and the Zeeman interaction $\left(\mathbf{H}_{\mathrm{Z}}\right)$. For the theoretical description of the crystal field states, we will use the so-called trigonal coordinate system $^{1}$ with the $Z$ axis directed along the $C_{3}$ axis (Figure 5). Trigonal one-electron states (complex trigonal basis) related to this coordinate system are expressed in terms of the d-functions $\xi=y z, \eta=x z, \zeta=x y\left(\mathrm{t}_{2 \mathrm{~g}}\right), u=3 z^{2}-r^{2}$, and $v=\sqrt{3}\left(x^{2}-\right.$ $\left.y^{2}\right)\left(\mathrm{e}_{\mathrm{g}}\right)$ defined in the tetragonal basis as follows: ${ }^{1}$

$$
\begin{aligned}
& \left\{\begin{array}{l}
\left|x_{+}\right\rangle=-(1 / \sqrt{3})\left(\epsilon \xi+\epsilon^{*} \eta+\zeta\right) \\
\left|x_{-}\right\rangle=(1 / \sqrt{3})\left(\epsilon^{*} \xi+\epsilon \eta+\zeta\right) \\
\left|x_{0}\right\rangle=(1 / \sqrt{3})(\xi+\eta+\zeta)
\end{array}\right. \\
& \left\{\begin{array}{l}
u_{+}=-(1 / \sqrt{2})(u+i v) \\
u_{-}=(1 / \sqrt{2})(u-i v)
\end{array} \epsilon=\exp (2 \pi i / 3)\right.
\end{aligned}
$$

For brevity, hereafter the parity label $g$ will be left out in the notations of the irreducible representations of the $O_{h}$-group. 
As mentioned, the intermediate coupling scheme used, in a strict sense, by $\mathrm{Liehr}^{8}$ seems to be appropriate for $5 \mathrm{~d}$ ions. In this scheme, the strong spin-orbit coupling is employed at the first stage of the calculation when constructing the one-electron orbitals. Here, however, we prefer to employ an alternative route based on the strong crystal field scheme with subsequent allowance for mixing of the appropriate terms by the spinorbit interaction. This approach to the problem has, at the very least, the following advantages. First, the theoretical footing for the strong crystal field scheme is well developed and the main matrix elements (energy matrices, spin-orbit interactions) are tabulated. ${ }^{1,2}$ Second, the truncated basis set that provides a satisfactory accuracy to the results can be chosen by the use of the Tanabe-Sugano diagrams. Finally, a strong field basis set is convenient for the consideration of the low-symmetry crystal fields that can be represented by the irreducible tensor operators in the $O_{h}$-group.

The basis for the $\mathrm{d}^{5}$ shell consists of the strong cubic crystal field states related to the trigonal coordinate system

$$
\left|\mathrm{t}_{2}^{n}\left(\mathrm{~S}_{1} \Gamma_{1}\right) \mathrm{e}^{m}\left(\mathrm{~S}_{2} \Gamma_{2}\right) \mathrm{S} \Gamma M_{\mathrm{S}} M\right\rangle
$$

where $\Gamma$ is an irreducible representation of $O_{h}$-group and $M$ enumerates the basis functions of this representation, namely, $M=x_{ \pm}\left(a_{ \pm}\right), x_{0}\left(a_{0}\right)$ for $\Gamma=T_{2}\left(T_{1}\right)$ and $M=u_{ \pm}$for $\Gamma=E$ (we use the notations of Sugano et al. $\left.{ }^{1}\right), S_{1} \Gamma_{1}$ designates the state of $\mathrm{t}_{2}^{n}$ subshell, $\mathrm{S}_{2} \Gamma_{2}$ is that for $\mathrm{e}^{m}$ subshell. The one-electron states $x_{+}$and $x_{-}$form the $E$ basis in $C_{3 v}$, as well as $u_{+}$and $u_{-}$, while function $x_{0}$ belongs to $A_{1}$. The same notations are assumed for the many-electron basis, eq 3 .

In the basis given by eq 3 , the cubic crystal field operator is represented by the diagonal matrix:

$$
\begin{array}{r}
\left\langle\mathrm{t}_{2}^{n}\left(\mathrm{~S}_{1} \Gamma_{1}\right) \mathrm{e}^{m}\left(\mathrm{~S}_{2} \Gamma_{2}\right) \mathrm{S} \Gamma M_{\mathrm{S}} M\left|\mathbf{V}_{\mathrm{C}}\right| \mathrm{t}_{2}^{n^{\prime}}\left(\mathrm{S}_{1}^{\prime} \Gamma_{1}^{\prime}\right) \mathrm{e}^{m^{\prime}}\left(\mathrm{S}_{2}^{\prime} \Gamma_{2}^{\prime}\right) \mathrm{S}^{\prime} \Gamma^{\prime} M_{\mathrm{S}^{\prime}}^{\prime} M^{\prime}\right\rangle= \\
(-4 n+6 m) \times \\
D q \delta_{n n^{\prime}} \delta_{m m^{\prime}} \delta_{\mathrm{S}_{1} \mathrm{~S}_{1}^{\prime}} \delta_{\mathrm{S}_{2} \mathrm{~S}_{2}^{\prime}} \delta_{\Gamma_{1} \Gamma_{1}^{\prime}{ }_{1}} \delta_{\Gamma_{2} \Gamma_{2}^{\prime}} \delta_{\mathrm{SS}^{\prime}} \delta_{\mathrm{M}_{\mathrm{S}} \mathrm{M}_{\mathrm{S}}^{\prime}} \delta_{\Gamma \Gamma^{\prime}} \delta_{\mathrm{MM}^{\prime}}
\end{array}
$$

For the $\mathrm{d}^{5}$ shell of $\operatorname{Re}(\mathrm{II})$ ion, $n+m=n^{\prime}+m^{\prime}=5$. In eq 4 , the cubic crystal field splitting of the one-electron levels is usually defined as

$$
\begin{aligned}
\left\langle\mathrm{t}_{2} x_{ \pm}\left|\mathbf{V}_{\mathrm{c}}\right| \mathrm{t}_{2} x_{ \pm}\right\rangle=\left\langle\mathrm{t}_{2} x_{0}\left|\mathbf{V}_{\mathrm{c}}\right| \mathrm{t}_{2} x_{0}\right\rangle=- & 4 D q \\
& \left\langle\mathrm{e} u_{ \pm}\left|\mathbf{V}_{\mathrm{c}}\right| \mathrm{e} u_{ \pm}\right\rangle=6 D q
\end{aligned}
$$

so that one-electron $t_{2}$ and e levels are separated by the gap $10 D q$.

The matrix elements of the interelectronic interaction Hamiltonian $\mathbf{V}_{\text {ee }}$ between the states (eq 3 ) are expressed in terms of Racah parameters $B$ and $C$, and the energy matrices are given by Sugano et al. ${ }^{1}$ To avoid overparametrization in the best fit procedure, we will use the following values of these parameters calculated for the free $\operatorname{Re}(\mathrm{II})$ ion: $^{6} B=647 \mathrm{~cm}^{-1}, C / B=4.27$.

3.2. Trigonal Crystal Field. In calculation of the matrices of the trigonal field and spin-orbit interaction, we shall use the methods developed in ref 1 . Because $x_{0}$ belongs to $A_{1}$ in $C_{3 v}$, the trigonal crystal field operator can be represented as the irreducible tensor operator of the type $\mathbf{V}_{\mathrm{T}_{2} x_{0}}$ of the $O_{h}$ group, so one can apply the Wigner-Eckart theorem to build the matrix of this operator. The nonvanishing matrix elements of $\mathbf{V}_{\mathrm{T}_{2} x_{0}}$ within the one-electron states, eq 2, are the following:

$$
\begin{aligned}
&\left\langle x_{ \pm}\left|\mathbf{V}_{\mathrm{T}_{2} x_{0}}\right| x_{ \pm}\right\rangle=-\frac{1}{3 \sqrt{2}}\left\langle\mathrm{t}_{2}|| \mathbf{V}_{\mathrm{T}_{2}}|| \mathrm{t}_{2}\right\rangle \\
&\left\langle x_{0}\left|\mathbf{V}_{\mathrm{T}_{2} x_{0}}\right| x_{0}\right\rangle=\frac{2}{3 \sqrt{2}}\left\langle\mathrm{t}_{2}|| \mathbf{V}_{\mathrm{T}_{2}}|| \mathrm{t}_{2}\right\rangle \\
&\left\langle x_{ \pm}\left|\mathbf{V}_{\mathrm{T}_{2} x_{0}}\right| u_{ \pm}\right\rangle=\frac{1}{\sqrt{6}}\left\langle\mathrm{t}_{2}|| \mathbf{V}_{\mathrm{T}_{2}}|| \mathrm{e}\right\rangle
\end{aligned}
$$

The first two matrix elements represent one-electron trigonal traceless splitting of the cubic $t_{2}$ level into e and $a_{1}$, and the remaining matrix element is responsible for the mixing of two trigonal doublets, e. The reduced matrix elements $\left\langle\mathrm{t}_{2}|| \mathbf{V}_{\mathrm{T}_{2}}|| \mathrm{t}_{2}\right\rangle$ and $\left\langle\mathrm{t}_{2}|| \mathbf{V}_{\mathrm{T}_{2}} \mid \mathrm{e}\right\rangle$ can be regarded as the trigonal crystal field parameters. Trigonal field parameters $v$ and $v^{\prime}$ introduced by Pryce and Runciman ${ }^{15}$ will also be employed. These parameters can be expressed in terms of the reduced matrix elements in eq 6

$$
v=\frac{1}{\sqrt{2}}\left\langle\mathrm{t}_{2}|| \mathbf{V}_{\mathrm{T}_{2}}|| \mathrm{t}_{2}\right\rangle, \quad v^{\prime}=\frac{1}{\sqrt{6}}\left\langle\mathrm{t}_{2}|| \mathbf{V}_{\mathrm{T}_{2}}|| \mathrm{e}\right\rangle
$$

so eq 6 can be rewritten in the conventional form:

$$
\begin{aligned}
\left\langle x_{ \pm}\left|\mathbf{V}_{\mathrm{T}_{2} x_{0}}\right| x_{ \pm}\right\rangle=-\frac{1}{3} v, \quad\left\langle x_{0}\left|\mathbf{V}_{\mathrm{T}_{2} x_{0}}\right| x_{0}\right\rangle=\frac{2}{3} v & \\
& \left\langle x_{ \pm}\left|\mathbf{V}_{\mathrm{T}_{2} x_{0}}\right| u_{ \pm}\right\rangle=v^{\prime}
\end{aligned}
$$

Three parameters, $D q, v$, and $v^{\prime}$, provide a complete description of the crystal field of $C_{3 v}$ symmetry acting on the $\operatorname{Re}(\mathrm{II})$ ion. The matrix elements of the operator $\mathbf{V}_{\mathrm{T}_{2} x_{0}}$ between the states, eq 1, can be calculated with the aid of the Wigner-Eckart theorem, and the problem is reduced to the calculation of the following reduced matrix elements:

$$
\left\langle\mathrm{t}_{2}^{n}\left(\mathrm{~S}_{1} \Gamma_{1}\right) \mathrm{e}^{m}\left(\mathrm{~S}_{2} \Gamma_{2}\right) \mathrm{S} \Gamma|| \mathbf{V}_{\mathrm{T}_{2}} \| \mathrm{t}_{2}^{n-k}\left(\mathrm{~S}_{1}^{\prime} \Gamma_{1}^{\prime}\right) \mathrm{e}^{m+k}\left(\mathrm{~S}_{2}^{\prime} \Gamma_{2}^{\prime}\right) \mathrm{S}^{\prime} \Gamma^{\prime}\right\rangle
$$

These many-electron matrix elements vanish if $|k| \geq 2$, and for $|k|=0$ and $|k|=1$, they can be expressed in terms of the oneelectron reduced matrix elements as follows:

$$
\begin{aligned}
& \left\langle\mathrm{t}_{2}^{n}\left(\mathrm{~S}_{1} \Gamma_{1}\right) \mathrm{e}^{m}\left(\mathrm{~S}_{2} \Gamma_{2}\right) \mathrm{S} \Gamma|| \mathbf{V}_{\mathrm{T}_{2}} \| \mathrm{t}_{2}^{n}\left(\mathrm{~S}_{1}^{\prime} \Gamma_{1}^{\prime}\right) \mathrm{e}^{m}\left(\mathrm{~S}_{2}^{\prime} \Gamma_{2}^{\prime}\right) \mathrm{S}^{\prime} \Gamma^{\prime}\right\rangle= \\
& C_{1}\left(\mathrm{~S}_{1} \Gamma_{1} \mathrm{~S}_{2} \Gamma_{2} \Gamma_{1}^{\prime} \Gamma_{2}^{\prime} \Gamma \Gamma^{\prime}\right)\left\langle\mathrm{t}_{2}|| \mathbf{V}_{\mathrm{T}_{2}}|| \mathrm{t}_{2}\right\rangle \delta_{\mathrm{S}_{1} \mathrm{~S}_{1}^{\prime}} \delta_{\mathrm{S}_{2} \mathrm{~S}_{2}^{\prime}} \delta_{\Gamma_{2} \Gamma_{2}^{\prime}} \delta_{\mathrm{SS}^{\prime}}, \\
& \left\langle\mathrm{t}_{2}^{n}\left(\mathrm{~S}_{1} \Gamma_{1}\right) \mathrm{e}^{m}\left(\mathrm{~S}_{2} \Gamma_{2}\right) \mathrm{S} \Gamma|| \mathbf{V}_{\mathrm{T}_{2}}|| \mathrm{t}_{2}^{n-1}\left(\mathrm{~S}_{1}^{\prime} \Gamma_{1}^{\prime}\right) \mathrm{e}^{m+1}\left(\mathrm{~S}_{2}^{\prime} \Gamma_{2}^{\prime}\right) \mathrm{S}^{\prime} \Gamma^{\prime}\right\rangle= \\
& C_{2}\left(\mathrm{~S}_{1} \Gamma_{1} \mathrm{~S}_{2} \Gamma_{2} \mathrm{~S}_{1}^{\prime} \Gamma_{1}^{\prime} \mathrm{S}_{2}^{\prime} \Gamma_{2}^{\prime} \Gamma \Gamma^{\prime}\right)\left\langle\mathrm{t}_{2}|| \mathbf{V}_{\mathrm{T}_{2}}|| \mathrm{e}\right\rangle \delta_{\mathrm{SS}^{\prime}}
\end{aligned}
$$

To express the many-electron reduced matrix elements in terms of the one-electron reduced matrix elements and consequently in terms of the parameters $v$ and $v^{\prime}$, one must calculate selected matrix elements of $\mathbf{V}_{\mathrm{T}_{2} x_{0}}$ using the explicit form of the wave functions of the type 3 expressed in terms of Slater determinants. These functions are given in Appendix I. Matrix elements of the trigonal field operator expressed through the parameters $v$ and $v^{\prime}$ are given in Appendix II.

3.3. Spin-Orbit Interaction. The Hamiltonian of the spinorbit interaction

$$
\mathbf{H}_{\mathrm{SO}}=\sum_{i} \boldsymbol{\xi}\left(r_{i}\right) \mathbf{l}_{i} \mathbf{s}_{i}
$$

can be represented as a linear combination of the irreducible double tensor operators:

$$
\mathbf{H}_{\mathrm{SO}}=\sum_{q=0, \pm 1}(-1)^{q} \mathbf{V}_{1 q \mathbf{T}_{1}-q}
$$


The expressions of $\mathbf{V}_{1 q \mathbf{T}_{1}-q}$ in terms of $\xi$ and operators $\mathbf{l}_{i}$ and $\mathbf{s}_{i}$ are given in ref 1 . The operators $\mathbf{V}_{1 q \mathbf{T}_{1}-q}$ are transformed as an irreducible tensor of the rank 1 in spin space and as a $\mathbf{T}_{1}$-type irreducible tensor in orbital space. In eq 11, the components $a_{+}, a_{-}$, and $a_{0}$ of $\mathbf{T}_{1}$ are labeled, respectively, by the projections $1,-1$, and 0 of the pseudo-angular momentum $\mathbf{I}=1$. The matrix elements of $\mathbf{H}_{\mathrm{SO}}$ can be calculated using the Wigner-Eckart theorem. Reduced matrix elements

$$
\left\langle\mathrm{t}_{2}^{n}\left(\mathrm{~S}_{1} \Gamma_{1}\right) \mathrm{e}^{m}\left(\mathrm{~S}_{2} \Gamma_{2}\right) \mathrm{S} \Gamma|| \mathbf{V}_{1 \mathbf{T}_{1}} \| \mathrm{t}_{2}^{n-k}\left(\mathrm{~S}_{1}^{\prime} \Gamma_{1}^{\prime}\right) \mathrm{e}^{m+k}\left(\mathrm{~S}_{2}^{\prime} \Gamma_{2}^{\prime}\right) \mathrm{S}^{\prime} \Gamma^{\prime}\right\rangle
$$

can be expressed in terms of the one-electron reduced matrix elements. In the $d$-function approximation, these matrix elements can be written as

$$
\left\langle\mathrm{t}_{2}|| \mathbf{V}_{1 \mathbf{T}_{1}}|| \mathrm{t}_{2}\right\rangle=3 i \kappa \xi, \quad\left\langle\mathrm{t}_{2}|| \mathbf{V}_{1 \mathbf{T}_{1}}|| \mathrm{e}\right\rangle=-3 \sqrt{2} i \kappa^{\prime} \zeta^{\prime}
$$

where $\zeta$ and $\zeta^{\prime}$ are the spin-orbit parameters and $\kappa$ and $\kappa^{\prime}$ are the corresponding orbital reduction factors. The reduced matrices, eq 12, for all $\mathrm{d}$ ions are given by Sugano et al. ${ }^{1}$ Neglecting the difference in radial functions for the $t_{2}$ and $e$ orbitals, we reduce the number of the spin-orbit parameters to one parameter, $\zeta^{\prime}=\zeta$, and in this approximation, $\kappa=\kappa^{\prime}$ as well. In the subsequent calculations, we will use the value of $\zeta=2100 \mathrm{~cm}^{-1}$ for the value of the $5 \mathrm{~d}$ orbitals of $\operatorname{Re}(\mathrm{II})$, which is in agreement with the estimated value $2,16,17$ of $\zeta$ for a free $\operatorname{Re}(\mathrm{II})$ ion. Much less is known about the orbital reduction factor for $\operatorname{Re}(\mathrm{II})$, so it seems to be reasonable to consider $\kappa$ as a fitting parameter.

3.4. Zeeman Interaction. Accounting for the fact that the spin operator is the first rank irreducible tensor and the orbital angular momentum operator is an irreducible tensor of $\mathbf{T}_{1}$-type in the $O_{h}$ group, one can present the operator of Zeeman interaction in the following form:

$$
\mathbf{H}_{\mathrm{Z}}=\beta\left(\kappa \mathbf{L}+g_{\mathrm{e}} \mathbf{S}\right) \mathbf{H}=\beta \sum_{q=0, \pm 1}(-1)^{q}\left(\kappa \mathbf{L}_{\mathbf{T}_{1}} q+g_{e} \mathbf{S}_{1 q}\right) H_{1-q}
$$

where $H_{1 \pm 1}=\mp(1 / \sqrt{2})\left(H_{X} \pm i H_{Y}\right)$ and $H_{10}=H_{Z}$ are the cyclic components of the applied magnetic field and $X, Y$, and $Z$ designate the trigonal axes. The approach to the evaluation of the reduced matrix elements

$$
\left\langle\mathrm{t}_{2}^{n}\left(\mathrm{~S}_{1} \Gamma_{1}\right) \mathrm{e}^{m}\left(\mathrm{~S}_{2} \Gamma_{2}\right) \mathrm{S} \Gamma|| \mathbf{L}_{\mathbf{T}_{1}}|| \mathrm{t}_{2}^{n-k}\left(\mathrm{~S}_{1}^{\prime} \Gamma_{1}^{\prime}\right) \mathrm{e}^{m+k}\left(\mathrm{~S}_{2}^{\prime} \Gamma_{2}^{\prime}\right) \mathrm{S}^{\prime} \Gamma^{\prime}\right\rangle
$$

of the angular momentum operator is developed in ref 1 . These many-electron matrix elements vanish if $|k| \geq 2$. For $|k|=0$, one can find

$$
\begin{aligned}
& \left\langle\mathrm{t}_{2}^{n}\left(\mathrm{~S}_{1} \Gamma_{1}\right) \mathrm{e}^{m}\left(\mathrm{~S}_{2} \Gamma_{2}\right) \mathrm{S} \Gamma|| \mathbf{L}_{\mathbf{T}_{1}}|| \mathrm{t}_{2}^{n}\left(\mathrm{~S}_{1}^{\prime} \Gamma_{1}^{\prime}\right) \mathrm{e}^{m}\left(\mathrm{~S}_{2}^{\prime} \Gamma_{2}^{\prime}\right) \mathrm{S}^{\prime} \Gamma^{\prime}\right\rangle= \\
& C_{1}\left(\mathrm{~S}_{1} \Gamma_{1} \mathrm{~S}_{2} \Gamma_{2} \Gamma_{1}^{\prime} \Gamma_{2}^{\prime} \Gamma \Gamma^{\prime}\right)\left\langle\mathrm{t}_{2} \| \mathbf{l}_{\mathrm{T}_{1}}|| \mathrm{t}_{2}\right\rangle \delta_{\mathrm{S}_{1} \mathrm{~S}_{1}^{\prime}} \delta_{\mathrm{S}_{2} \mathrm{~S}_{2}^{\prime}} \delta_{\Gamma_{2} \Gamma_{2}^{\prime}} \delta_{\mathrm{SS}^{\prime}}
\end{aligned}
$$

In the case of $|k|=1$, we obtain

$$
\begin{array}{r}
\left\langle\mathrm{t}_{2}^{n}\left(\mathrm{~S}_{1} \Gamma_{1}\right) \mathrm{e}^{m}\left(\mathrm{~S}_{2} \Gamma_{2}\right) \mathrm{S} \Gamma|| \mathbf{L}_{\mathbf{T}_{1}}|| \mathrm{t}_{2}^{n-1}\left(\mathrm{~S}_{1}^{\prime} \Gamma_{1}^{\prime}\right) \mathrm{e}^{m+1}\left(\mathrm{~S}_{2}^{\prime} \Gamma_{2}^{\prime}\right) \mathrm{S}^{\prime} \Gamma^{\prime}\right\rangle= \\
C_{2}\left(\mathrm{~S}_{1} \Gamma_{1} \mathrm{~S}_{2} \Gamma_{2} \mathrm{~S}_{1}^{\prime} \Gamma_{1}^{\prime} \mathrm{S}_{2}^{\prime} \Gamma_{2}^{\prime} \Gamma \Gamma^{\prime}\right)\left\langle\mathrm{t}_{2}\left\|\mathbf{l}_{\mathbf{T}_{1}}\right\| \mathrm{e}\right\rangle \delta_{\mathrm{SS}^{\prime}}
\end{array}
$$

The results of the calculations of these matrix elements within the adopted basis set are given in Appendix III.

Spin-operators in eq 14 act within each crystal field multiplet, eq 3 , so the reduced matrix elements of spin-tensor $\mathbf{S}_{1 q}$ (operating on the spin variables solely) is diagonal with respect to all crystal field quantum numbers and can be written with aid of the general definition for the reduced matrix element of the first rank tensor ${ }^{18}$ acting in spin space:

$$
\begin{aligned}
& \left\langle\mathrm{t}_{2}^{n}\left(\mathrm{~S}_{1} \Gamma_{1}\right) \mathrm{e}^{m}\left(\mathrm{~S}_{2} \Gamma_{2}\right) \mathrm{S} \Gamma|| \mathbf{S}_{1} \| \mathrm{t}_{2}^{n-k}\left(\mathrm{~S}_{1}^{\prime} \Gamma_{1}^{\prime}\right) \mathrm{e}^{m+k}\left(\mathrm{~S}_{2}^{\prime} \Gamma_{2}^{\prime}\right) \mathrm{S}^{\prime} \Gamma^{\prime}\right\rangle= \\
& {[S(S+1)(2 S+1)]^{1 / 2} \delta_{\mathrm{S}_{1} \mathrm{~S}_{1}^{\prime}} \delta_{\mathrm{S}_{2} \mathrm{~S}_{2}^{\prime}} \delta_{\Gamma_{1} \Gamma^{\prime}{ }_{1}} \delta_{\Gamma_{2} \Gamma_{2}^{\prime}} \delta_{\Gamma \Gamma^{\prime}} \delta_{\mathrm{SS}^{\prime}}}
\end{aligned}
$$

Finally, let us focus on the problem of the appropriate choice of the truncated basis set. This basis should include a sufficient number of states to reproduce the energies of the low-lying group of levels provided by the Tanabe-Sugano diagram for the $\mathrm{d}^{5}$ ion. To comply with the jj-coupling, the full range of the energy levels included in the basis should significantly exceed effective spin-orbit interaction. One can see that by providing a strong or moderate cubic crystal field, the low-lying group of levels that includes the eight terms $2^{2} \mathrm{~T}_{2},{ }^{6} \mathrm{~A}_{1},{ }^{4} \mathrm{~T}_{1},{ }^{4} \mathrm{~T}_{2},{ }^{2} \mathrm{~A}_{2},{ }^{2} \mathrm{~T}_{1}$, and ${ }^{2} \mathrm{E}_{2}$ are well separated from the excited ones. To properly reproduce the energies of these eight terms, the basis should contain a sufficient number of states. Inspecting the matrices of the Coulomb interaction ${ }^{1}$ and neglecting the high-energy states, we arrive at the following basis set including 82 states:

$$
\begin{aligned}
& \mathrm{t}_{2}^{5},{ }^{2} \mathrm{~T}_{2} ; \mathrm{t}_{2}^{4}\left({ }^{3} \mathrm{~T}_{1}\right) \mathrm{e},{ }^{2} \mathrm{~T}_{2} ; \mathrm{t}_{2}^{4}\left({ }^{3} \mathrm{~T}_{1}\right) \mathrm{e},{ }^{2} \mathrm{~T}_{2} ; \mathrm{t}_{2}^{3}\left({ }^{4} \mathrm{~A}_{2}\right) \mathrm{e}^{2}\left({ }^{3} \mathrm{~A}_{2}\right),{ }^{6} \mathrm{~A}_{1} ; \\
& \mathrm{t}_{2}^{4}\left({ }^{3} \mathrm{~T}_{1}\right) \mathrm{e},{ }^{4} \mathrm{~T}_{1} ; \mathrm{t}_{2}^{4}\left({ }^{3} \mathrm{~T}_{1}\right) \mathrm{e},{ }^{4} \mathrm{~T}_{2} ; \mathrm{t}_{2}^{3}\left({ }^{2} \mathrm{~T}_{2}\right) \mathrm{e}^{2}\left({ }^{3} \mathrm{~A}_{2}\right),{ }^{4} \mathrm{~T}_{2} ; \mathrm{t}_{2}^{4}\left({ }^{3} \mathrm{~T}_{1}\right) \mathrm{e},{ }^{2} \mathrm{~T}_{1} ; \\
& \mathrm{t}_{2}^{4}\left({ }^{1} \mathrm{~T}_{2}\right) \mathrm{e},{ }^{2} \mathrm{~T}_{1} ; \mathrm{t}_{2}^{4}\left({ }^{1} \mathrm{~A}_{1}\right) \mathrm{e},{ }^{2} \mathrm{E} ; \mathrm{t}_{2}{ }^{4}\left({ }^{1} \mathrm{E}\right) \mathrm{e},{ }^{2} \mathrm{E} ; \mathrm{t}_{2}^{4}\left({ }^{1} \mathrm{E}\right) \mathrm{e},{ }^{2} \mathrm{~A}_{2}(19)
\end{aligned}
$$

This basis allows us to reproduce the low energy section of the Tanabe-Sugano diagram in a strong or moderate cubic crystal field. There are several other electronic configurations that contribute to the ${ }^{2} \mathrm{~T}_{2},{ }^{4} \mathrm{~T}_{1},{ }^{4} \mathrm{~T}_{2},{ }^{2} \mathrm{~A}_{2},{ }^{2} \mathrm{~T}_{1}$, and ${ }^{2} \mathrm{E}$ terms. One can expect that the effect of mixing of these states with the remaining excited states originating from the excited electronic configurations is small. At the same time, the Coulomb mixing of these states with the states (eq 19) is weak because these states are much higher in energy. For this reason, they are not included in the basis set (eq 19).

\section{Discussion}

Due to the trigonal symmetry of the ligand environment of the $\operatorname{Re}(\mathrm{II})$ ions, the magnetic susceptibility tensor has two principal values, $\chi_{Z Z} \equiv \chi_{\|}$and $\chi_{X X}=\chi_{Y Y} \equiv \chi_{\perp}$, related to the $C_{3}$-axis. These principal values are calculated using the formula

$$
\chi_{\alpha \alpha}=N_{\mathrm{A}} k_{\mathrm{B}} T\left[\frac{\partial^{2}}{\partial H_{\alpha}^{2}} \ln Z\left(H_{\alpha}\right)\right]_{H \alpha \rightarrow 0}
$$

The partition function is given by

$$
Z\left(H_{\alpha}\right)=\sum_{i} \exp \left[-E_{i}\left(H_{\alpha}\right) /\left(k_{\mathrm{B}} T\right)\right]
$$

where $E_{i}\left(H_{\alpha}\right)$ are the energies of the system in the presence of the external magnetic field. The susceptibility for a powder sample is then calculated as follows:

$$
\chi=\left({ }^{1}{ }_{3}\right)\left(\chi_{\|}+2 \chi_{\perp}\right)
$$

The model that we have developed should actually be referred to as an approximate jj-coupling approach. When considering the model, the question arises: is the intricacy of the approach really necessary to describe the data? So, let us consider the results of a simpler model developed in our recent papers ${ }^{19,20}$ based on the true Russell-Saunders scheme in the limit of a strong cubic field approximation. In this model, the strong cubic 


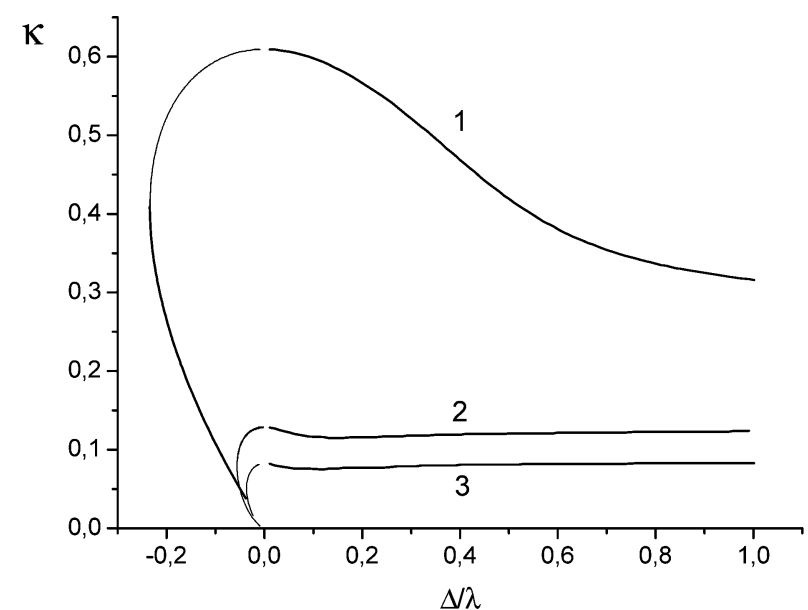

Figure 6. $(\kappa, \Delta / \lambda)$-diagram for the measured values $A=0.20541 \mathrm{~cm}^{3}$ $\mathrm{K} \mathrm{mol}^{-1}$ and $B=0.0013632 \mathrm{~cm}^{3} \mathrm{~mol}^{-1}$ (compound 2): (1) curve of constant parameter $A$ for $\lambda=-2000$ and $\lambda=-3000 \mathrm{~cm}^{-1}$; curves for constant parameter $B$ for $\lambda=(2)-2000$ and (3) $-3000 \mathrm{~cm}^{-1}$.

field ground term ${ }^{2} \mathrm{~T}_{2}\left(\mathrm{t}_{2}^{5}\right)$ is split by the spin-orbit interaction giving rise to a Kramers doublet $\Gamma_{7}$ (ground) and a quadruplet $\Gamma_{8}$, and then the trigonal field produces splitting of the excited quadruplet into two Kramers doublets: $\Gamma_{4}+\Gamma_{5}$ (complex conjugate representation of the double group $C_{3 v}$ ) and $\Gamma_{6}$; and mixing of the ground and excited states. The magnetic properties of the system in this approximation are determined by three parameters: spin-orbit interaction $(\lambda)$, orbital reduction $(\kappa)$, and the energy gap, $3 \Delta_{t} / 2$, determining the splitting of the cubic ${ }^{2} \mathrm{~T}_{2}\left(\mathrm{t}_{2}^{5}\right)$ term in the trigonal crystal field into an orbital singlet ${ }^{2} \mathrm{~A}_{1}$ and a doublet ${ }^{2} \mathrm{E}$. Using the analytical expression for $\chi T$ obtained within this model, ${ }^{19,20}$ we attempted to determine the parameters $\kappa$ and $\Delta_{t} / \lambda$ from the condition of least deviation of the calculated from experimental $\chi T$. The experimental dependence $\chi T$ vs $T$ can be described well enough by a straight line:

$$
\chi T=A+B T
$$

where, for instance, in compound 2 we found $A=0.20541 \mathrm{~cm}^{3}$ $\mathrm{K} \mathrm{mol}{ }^{-1}$, and $B=0.0013632 \mathrm{~cm}^{3} \mathrm{~mol}^{-1}$. Substituting these values of $A$ and $B$ into the theoretical expressions for $\chi T$, one can draw lines for the constant values of $A$ and $B$ in the plane of two variables $\kappa$ and $\Delta_{t} / \lambda$. These curves are presented in Figure 6 for two values of $\lambda,-2000$ and $-3000 \mathrm{~cm}^{-1}$, which cover the range of reasonable spin-orbit coupling parameters for $5 \mathrm{~d}$ ions. The parameter $A$ (which is independent of $\lambda$ ) is represented by the curve 1 for both values of $\lambda$, and curves 2 and 3 represent $B$ for these two values of $\lambda$, correspondingly. One can see that curve 3 lies lower than curve 1, but the curves corresponding to $A$ and $B$ do not intersect at any values of $\kappa$ and $\Delta_{t} / \lambda$, which means that the two parameters $\kappa$ and $\Delta_{t} / \lambda$ cannot be found to determine $A$ and $B$ satisfying experimental data. This result seems to be evidence of the fact that the model restricted by the Russel-Saunders scheme is unsuccessful for explaining the giant TIP and small $g$-factors of $\operatorname{Re}(\mathrm{II})$ in the title compounds.

After this realization, we proceed with the more general model described in the previous section. In this model, the energies that contribute to the partition function are obtained by means of the diagonalization of the Hamiltonian 1, defined in the basis shown in eq 19. Using fixed parameters $B=647 \mathrm{~cm}^{-1}, C / B=$ 4.27 , and $\zeta=2100 \mathrm{~cm}^{-1}$, we obtain the following sets of crystal field parameters and orbital reduction factors that provide the best fit for the $\chi T$ vs $T$ curves: $D q=1118 \mathrm{~cm}^{-1}, v=-12688$ $\mathrm{cm}^{-1}, v^{\prime}=10469 \mathrm{~cm}^{-1}$, and $\kappa=0.52$ for compound 1 and $D q=1133 \mathrm{~cm}^{-1}, v=-12021 \mathrm{~cm}^{-1}, v^{\prime}=10701 \mathrm{~cm}^{-1}$, and
Compound 1

Compound 2

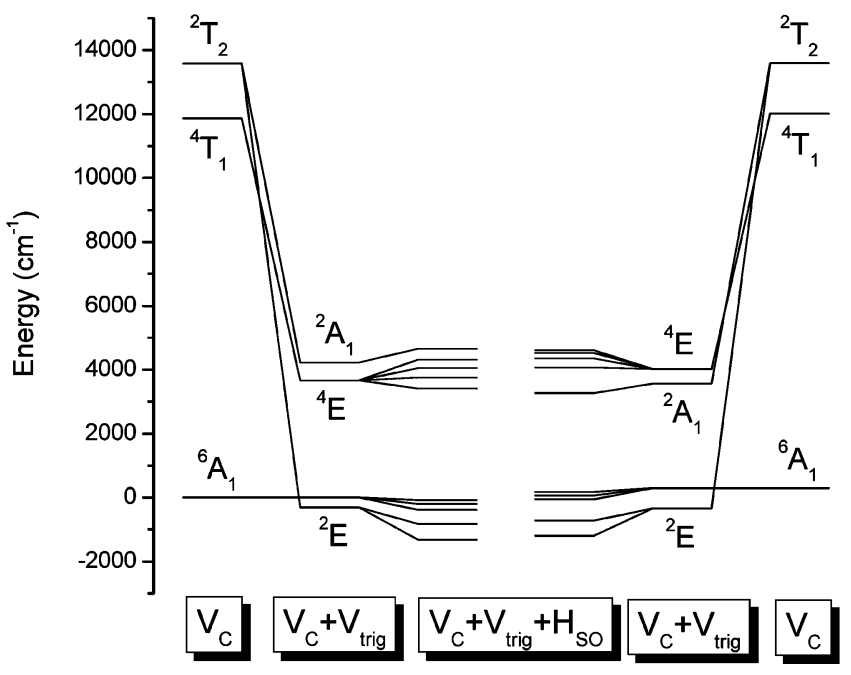

Figure 7. The splitting of the four lowest cubic crystal field terms by the trigonal crystal field and spin-orbit coupling calculated with the best-fit parameters.

$\kappa=0.49$ for compound 2 . Along with the parameters $v$ and $v^{\prime}$, the alternative trigonal field parameters $D \sigma$ and $D \tau$ are also commonly used in spectroscopy of metal complexes.,16 They are found to be the following: $D \sigma=-2418 \mathrm{~cm}^{-1}$, and $D \tau=$ $2991 \mathrm{~cm}^{-1}$ (compound 1); $D \sigma=-2607 \mathrm{~cm}^{-1}$, and $D \tau=2976$ $\mathrm{cm}^{-1}$ (compound 2). The agreement defined according to the least-squares criterion for

$$
(1 / N) \sum\left[(\chi T)_{\text {expt }}-(\chi T)_{\text {calcd }}\right]^{2} /(\chi T)_{\text {expt }}^{2}
$$

gave a relative error of $4.5 \times 10^{-5}$ for compound $\mathbf{1}$ and $9.1 \times$ $10^{-4}$ for compound 2 ( $N$ is the number of the experimental points). The obtained values of $\kappa$ are relatively small for both $5 \mathrm{~d}$ compounds, which exhibit a higher degree of covalency than that in analogous $3 \mathrm{~d}$ compounds. Figure 2 shows a perfect agreement between the calculated $\chi T$ vs $T$ dependence (solid lines) and the experimental ones. To clarify the meaning of the rather large values obtained for the trigonal crystal field parameters $v$ and $v^{\prime}$, one can evaluate the splitting of the oneelectron cubic state $\mathrm{t}_{2}\left(x_{0}, x_{ \pm}\right)$into the singlet $\left(x_{0}\right)$ and doublet $\left(x_{ \pm}\right)$by the trigonal crystal field. The energy gap $\Delta_{t}=\epsilon\left(x_{ \pm}\right)-$ $\epsilon\left(x_{0}\right)$ between the singlet and doublet is determined both by the diagonal matrix elements of $\mathbf{V}_{\text {trig }}$ between the $t_{2}$ states (these matrix elements relate to the parameter $v$ ) and the off-diagonal matrix elements connecting $\left|\mathrm{t}_{2} x_{ \pm}\right\rangle$states with the states $\left|\mathrm{e} u_{ \pm}\right\rangle$ that are $10 D q$ higher in energy (these matrix elements are expressed in terms of $v^{\prime}$ ). Solving the obtained $2 \times 2$ matrix with the best fit parameters $D q, v$, and $v^{\prime}$ given above, one obtains the following energy gaps: $\Delta_{t}=5132 \mathrm{~cm}^{-1}$ for compound 1 and $\Delta_{t}=4372 \mathrm{~cm}^{-1}$ for compound 2 . Comparing the gaps $\Delta_{t}$ with the corresponding cubic field splitting $10 D q$, one finds that for $1 \Delta_{t} /(10 D q) \approx 0.46$ and for $2 \Delta_{t} /(10 D q) \approx$ 0.39 . Both compounds are strongly noncubic, mainly because of the mixed ligand set surrounding the Re(II) ion. The scenario of the low-symmetry component of the crystal field being comparable to the cubic one is not extraordinary. A similar situation occurs in the reduced polyoxotungstate and polyoxomolybdate anions of Keggin and Dawson-Wells structures. ${ }^{5,21}$ In these systems, the noncubic crystal field produced by the oxygen atoms surrounding the $\mathrm{Mo}^{5+}$ and $\mathrm{W}^{5+}$ ions is comparable with the cubic field.

Figure 7 represents the low-lying section of the full spectrum $\left({ }^{6} \mathrm{~A}_{1},{ }^{2} \mathrm{~T}_{2}\right.$, and ${ }^{4} \mathrm{~T}_{1}$ terms split by the trigonal crystal field and 
spin-orbit interaction) for both compounds, obtained with the sets of the best fit parameters. Inspecting Figure 6 and the Tanabe-Sugano diagram for $\mathrm{d}^{5}$ ions, one can see that the cubic crystal field that provides the best fit proves to be relatively weak $(D q / B=1.73)$, so the ground state is indeed the highspin sextet ${ }^{6} \mathrm{~A}_{1}\left(\mathrm{t}_{2}^{3} \mathrm{e}^{2}\right)$ rather than the spin doublet ${ }^{2} \mathrm{~T}_{2}\left(\mathrm{t}_{2}^{5}\right)$ that would be expected in the case of a strong field. This result does not conform to the prevalent belief that $5 \mathrm{~d}$ complexes are always low-spin. After a survey of the literature, no representative was found on crystal fields in octahedral or distorted $\operatorname{Re}(\mathrm{II})$ complexes. In this view, we would like to cite $^{2}$ the data on the $D q$ values that are available for the trivalent heavy metal ions: $\mathrm{Mo}^{3+}\left(4 \mathrm{~d}^{3}\right) \mathrm{Dq}=2000 \mathrm{~cm}^{-1}$ and $\mathrm{W}^{3+}\left(5 \mathrm{~d}^{3}\right) \mathrm{Dq}=2800 \mathrm{~cm}^{-1}$. Taking into account that in systems of divalent ions the value of $D q$ is usually approximately half of that for trivalent ions, ${ }^{1}$ the obtained value of $D q$ for $\operatorname{Re}(\mathrm{II})$ appears to be unexpectedly low but nevertheless falls in the range of reasonable values. Furthermore, it should be noted that the mixed ligand complexes under consideration are far from ideal octahedral. In fact, the trigonal component of the crystal field is found to be comparable with the cubic one. Under this condition, different contributions to the overall crystal field are inseparable and can give rise to underestimated values for $D q$.

The low spin-orbit doublet ${ }^{2} \mathrm{E}$ becomes the ground state because of strong trigonal crystal field splitting of the ${ }^{2} \mathrm{~T}_{2}$ term that results in the crossover of the levels ${ }^{6} \mathrm{~A}_{1}$ and ${ }^{2} \mathrm{E}$. This observation shows that $\operatorname{Re}(\mathrm{II})$ is, in fact, low-spin because of strong trigonal field even under the condition of relatively weak cubic field. The fine structure of the ground ${ }^{2} \mathrm{E}$ level (irrespective of the structure of the basis functions) can be considered in the pseudo-angular momentum representation in which the cubic orbital triplet ${ }^{2} \mathrm{~T}_{2}$ is associated with the pseudo-angular momentum $L=1$, so the full angular momentum $J$ takes on the values $J=1 / 2$ and $3 / 2$. In the axial crystal field, two Kramers doublets originating from spin-orbit splitting of ${ }^{2} \mathrm{E}$ correspond to $\left|M_{J}\right|=1 / 2(J=1 / 2,3 / 2)$ and $\left|M_{J}\right|=3 / 2(J=3 / 2)$. In the case under consideration, the $\left|M_{J}\right|=1 / 2$ doublet proves to be the ground sublevel, so one expects that the EPR spectra of these $\operatorname{Re}(\mathrm{II})$ compounds can be observed at low temperature. Within the strong cubic field approximation, spin-orbit splitting of ${ }^{2} \mathrm{E}$ is $\kappa \lambda$ (see Figure 1 in ref 20), and this value is reduced by the trigonal field with respect to its initial value $3 \lambda / 2$ in a cubic ${ }^{2} \mathrm{~T}_{2}$ term. ${ }^{19,20}$ As we shall see, the effect of mixing leads to reduction of this gap, which is closely related to the observed giant TIP.

The level ${ }^{2} \mathrm{E}$ is close to the first excited level ${ }^{6} \mathrm{~A}_{1}$ (the energy separation is around $311.6 \mathrm{~cm}^{-1}$ for $\mathbf{1}$ and around $645.2 \mathrm{~cm}^{-1}$ for 2). This proximity of the excited states is responsible for the giant TIP of these systems. Then, the trigonal terms ${ }^{2} \mathrm{E},{ }^{6} \mathrm{~A}_{1}$, and ${ }^{4} \mathrm{E}$ undergo spin-orbit splitting into Kramers doublets (see central part of Figure 7, the irreducible representations for the Kramers doublets are not shown). The spin-orbit splitting is small as compared to the trigonal crystal field splitting because of the strong covalence (small $\kappa$ values).

The energy gaps between the ground and first excited Kramers doublets in both compounds are close: $490 \mathrm{~cm}^{-1}$ for 1 and $471 \mathrm{~cm}^{-1}$ for 2 . At the same time, the energy separations between these two Kramers doublets and those arising from the splitting of the ${ }^{6} \mathrm{~A}_{1}$ term for compound $\mathbf{1}$ are approximately 2 times smaller than those for 2 (Figure 7). As a result, the wave functions of two low-lying Kramers doublets for compound 1 contain a more significant weight from the ${ }^{6} \mathrm{~A}_{1}$ states. In fact, for compound $\mathbf{1}$, the total weight of the ${ }^{6} \mathrm{~A}_{1}$ states in the wave function of the ground Kramers doublet is characterized by the value $\rho_{1}\left({ }^{6} \mathrm{~A}_{1}\right)=\sum_{M_{\mathrm{S}}}\left|C_{1}\left({ }^{6} \mathrm{~A}_{1}, M_{\mathrm{S}}\right)\right|^{2} \approx 0.13$, while

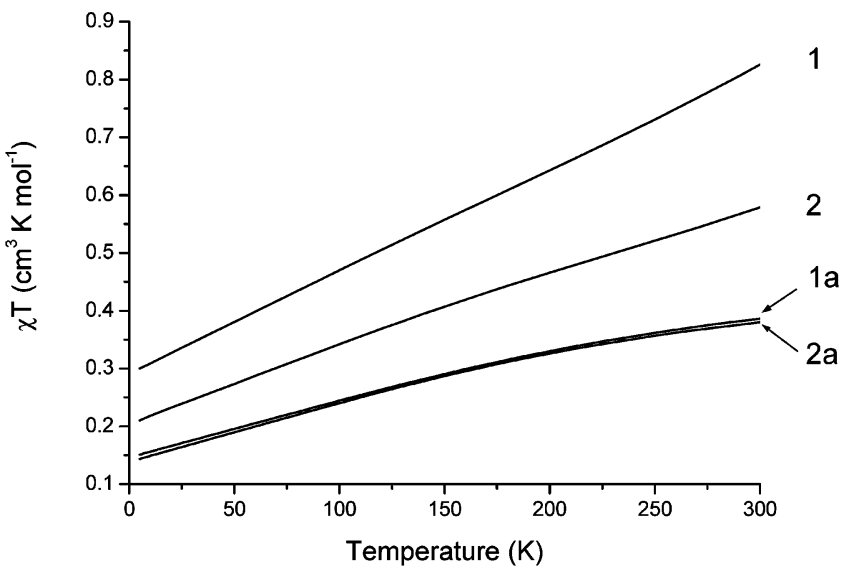

Figure 8. The influence of the ${ }^{6} \mathrm{~A}_{1}$ term on the $\chi T$ vs $T$ dependence. $\chi T$ vs $T$ curves were calculated with the best fit parameters (1) for compound 1 with the full basis, (2) for compound 2 with the full basis, (1a) for compound $\mathbf{1}$ with the reduced basis $\left({ }^{6} \mathrm{~A}_{1}\right.$ states are excluded), and ( $2 \mathrm{~b}$ ) for compound $\mathbf{2}$ with the reduced basis.

for the first excited Kramers doublet, this value is even higher, $\rho_{2}\left({ }^{6} \mathrm{~A}_{1}\right) \approx 0.2$. In compound 2 , these values are much smaller $\left(\rho_{1}\left({ }^{6} \mathrm{~A}_{1}\right) \approx 0.06, \rho_{2}\left({ }^{6} \mathrm{~A}_{1}\right) \approx 0.08\right)$, which accounts for the smaller values of the low-temperature effective magnetic moment and TIP (Figure 2). In fact, the more significant weight of the ${ }^{6} \mathrm{~A}_{1^{-}}$ state in both low-lying Kramers doublets promotes their efficient Zeeman mixing, thus increasing the large TIP values. The role of the ${ }^{6} \mathrm{~A}_{1}$-state can also be demonstrated by means of the numerical simulation of the dependence of $\chi T$ vs $T$ in the case when this state is excluded from the basis set (eq 19). Figure 8 shows that this exclusion leads to the appreciable decrease of $\chi T$ in such a way that the $\chi T$ vs T curves become nearly identical for both compounds. The small difference between the curves for $\mathbf{1}$ and $\mathbf{2}$ in Figure 8 is due only to the influence of the ${ }^{4} \mathrm{~T}_{1}$ term, which gives rise to the second excited group of Kramers doublets. Therefore, we arrive at the conclusion that the splitting and mixing of the three low-lying cubic terms ${ }^{2} \mathrm{~T}_{2},{ }^{6} \mathrm{~A}_{1}$, and ${ }^{4} \mathrm{~T}_{1}$ by the strong trigonal crystal field and spin-orbit coupling are of crucial importance for the understanding of the unusual magnetic behavior of the rhenium(II) complexes under study. One can observe a noticeable deviation of $\chi T$ vs $T$ from a straight line for compound 2 ; this can be attributed to the population of the low-lying levels so that the Van Vleck paramagnetism cannot be referred to as temperature-independent to a full extent. This can be considered as indirect evidence for the obtained energy pattern containing a closely spaced set of the low-lying levels.

Because the magnetic measurements were made on powder samples, the experimental data regarding the magnetic anisotropy are presently unavailable. So, at this stage of our study of $\operatorname{Re}(\mathrm{II})$ complexes, we can only make some predictions that follow from the theoretical considerations. Figure 9 shows $\chi_{\|} T$ and $\chi_{\perp} T$ calculated with the sets of best fit parameters. It is clear that both compounds are strongly anisotropic with a $C_{3}$ easy axis of magnetization that is peculiar to the magnetic behavior of the Kramers doublet systems carrying strong contribution of the orbital angular momentum. For example, for $1,\left(\chi_{\|} T\right)_{T \rightarrow 0}=0.8 \mathrm{~cm}^{3} \mathrm{~K} \mathrm{~mol}^{-1}$ and $\left(\chi_{\perp} T\right)_{T \rightarrow 0}=0.03 \mathrm{~cm}^{3} \mathrm{~K}$ $\mathrm{mol}^{-1}$; the degree of the low-temperature magnetic anisotropy defined as $\left(\chi_{\| T \rightarrow 0}-\chi_{\perp T \rightarrow 0}\right) / \chi_{T \rightarrow 0}$ is also very high: 2.57 for $\mathbf{1}$ and 2.38 for $\mathbf{2}$. The model also predicts strongly anisotropic $g$-factors that are closely related to the strong orbital contributions to the ground Kramers doublet $\left|M_{J}\right|=1 / 2$ in both compounds: $g_{\|}=2.9, g_{\perp}=0.7$ (compound 1) and $g_{\|}=2.4$, 

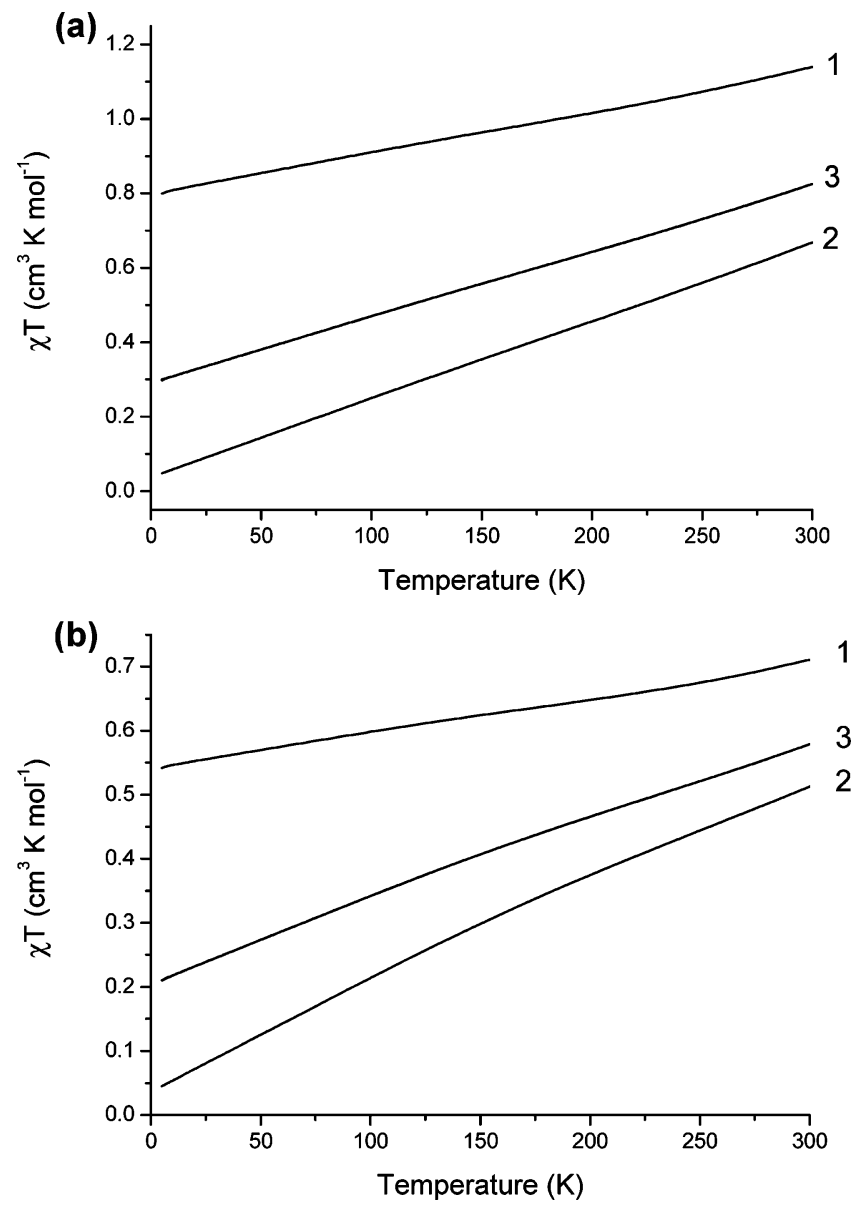

Figure 9. Anisotropic contributions to $\chi T$ calculated with the best fit parameters for (a) compound 1 and (b) compound 2: (1) $\chi_{\|} T$; (2) $\chi_{\perp} T$; (3) powder $\chi T$.

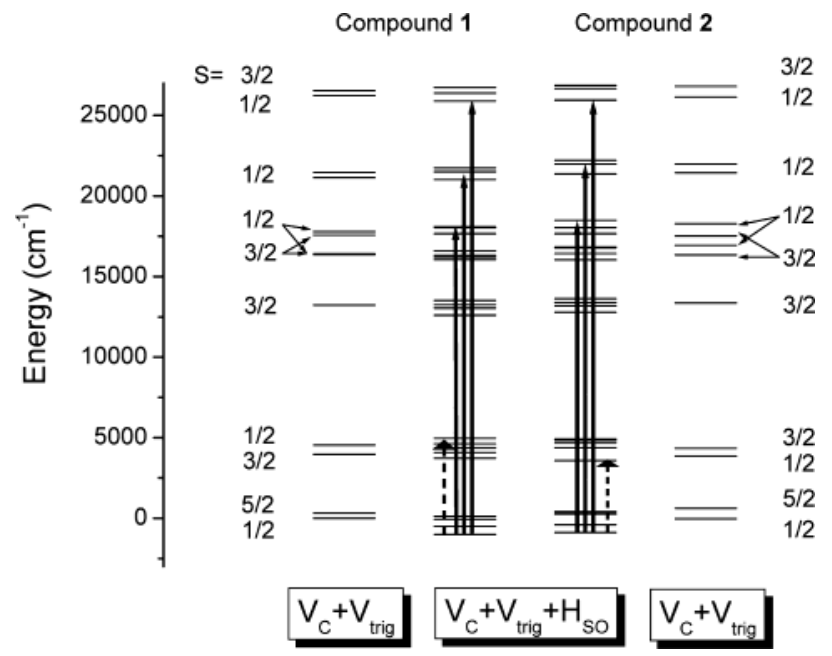

Figure 10. The scheme of spin-allowed transitions responsible for the observed and predicted electronic absorption bands.

$g_{\perp}=0.7$ (compound 2). The derived conclusions can be used for further justification of the model providing that the measurements of EPR and magnetic susceptibility on the single-crystal samples are available.

Unfortunately, as yet we do not have single-crystal EPR and magnetic susceptibility data that can provide rigorous support for justifying the model. In this situation, we address the results of our measurements of absorption and diffuse reflectance in a wide spectral area, from 200 to $2600 \mathrm{~nm}$. Figure 10 shows the energy patterns for compounds $\mathbf{1}$ and $\mathbf{2}$ calculated with the parameters providing the best fit to the magnetic data. The energy levels with and without allowance of spin-orbit interactions are presented. The transitions that are responsible for the most intense broad absorption band in the range $350-500 \mathrm{~nm}$ are also shown. These transitions occur from the ground Kramers doublet to the excited Kramers doublets that appear as a result of the spin-orbit splitting of the trigonal terms with $S=1 / 2$. Because this splitting is small compared to that produced by the trigonal crystal field, we can regard the relevant optical transitions as the spin-allowed ones. It is noted that the transition energies are higher for compound $\mathbf{2}$, which accounts for the observation that the maximum of the absorption band for this compound is blue-shifted with respect to the maximum for compound 1. Both the observed positions of these maxima and the half-widths of the bands are in qualitative agreement with the scheme of the transitions (Figure 10) based on the parameters obtained by the best-fit procedure for the magnetic data.

It should be mentioned that the calculations also predict the presence of electronic transitions in the infrared region between 4000 and $6000 \mathrm{~cm}^{-1}$ for both compounds. A band in the same wavelength range was observed in $\mathrm{K}_{2} \mathrm{IrX}_{6}(\mathrm{X}=\mathrm{Cl}, \mathrm{Br}, \mathrm{I}){ }^{22}$ The band formed by these lines can be attributed to the transitions between the ground Kramers doublet and the set of Kramers doublets originating from the trigonal ${ }^{2} \mathrm{~A}_{1}$ and ${ }^{4} \mathrm{E}$ terms mixed by spin-orbit interaction. These transitions are depicted in Figure 10 by the dashed arrows. The observed diffuse reflectance spectra for both compounds (Figure 4) show a series of transitions just in this spectral area. It is remarkable that the energy pattern obtained predicts a general shift of the lines in the NIR region for compound $\mathbf{1}$ that correspond very well to the experimental data. The calculated pattern also predicts a gap between the set of the low-lying levels ${ }^{2} \mathrm{E},{ }^{2} \mathrm{~A}_{1},{ }^{4} \mathrm{E}$, and the excited states, $5000-12500 \mathrm{~cm}^{-1}$. The gaps are approximately the same for both compounds and can be considered as an origin of the observed plateau in the diffuse reflectance spectra in just this region. For a detailed description of the shape of the electronic absorption bands and accurate assignment of the complicated picture of the observed transitions in the absorption and diffuse reflectance spectra, the vibronic interactions must be included in the model. Nevertheless, the brief discussion of this issue presented here along with the detailed discussion of the magnetic data indicate that the proposed model has a tangible ground. More comprehensive considerations of the optical and magnetic properties also require the allowance for a Jahn-Teller interaction in the ground manifold, as well as in the excited states, leading to a rather complicated vibronic problem. In our previous work, ${ }^{19,20}$ we considered the manifestations of the Jahn-Teller interaction in the limit of a strong cubic crystal field. To avoid overparametrization of the theory, we did not include this interaction in the present consideration. It should also be noted that the detailed study of the single-crystal samples could be of great significance in the study of the title Re(II) compounds.

\section{Concluding Remarks}

In this article, we present magnetic data for two complexes of $\operatorname{Re}(\mathrm{II})$ : $\left[\mathrm{Re}(\right.$ triphos $\left.)\left(\mathrm{CH}_{3} \mathrm{CN}\right)_{3}\right]\left[\mathrm{BF}_{4}\right]_{2}$ and $\left[\mathrm{Et}_{4} \mathrm{~N}\right][\operatorname{Re}($ triphos)$\left.(\mathrm{CN})_{3}\right]$. We developed a model that can be referred to as an approximate $\mathrm{jj}$-coupling scheme, which accounts for a strong spin-orbit interaction and a trigonal field operating in the truncated basis of strong cubic crystal field terms. The proposed model allowed us to elucidate the origin of the anomalously strong Van Vleck paramagnetism and relatively small $g$-factors for the ground state. Beyond simply fitting the $\chi T$ vs $T$ data, 
the energy pattern of $\operatorname{Re}(\mathrm{II})$ obtained with best fit parameters provides a qualitative explanation of the optical absorption bands observed in the range $350-550 \mathrm{~nm}$ and diffuse reflectance spectra in a wide range $200-2600 \mathrm{~nm}$, which can be considered as an additional argument justifying the model. We have also shown that the LS-coupling approach in a strong crystal field limit cannot explain the unusual magnetic behavior of the $\operatorname{Re}(\mathrm{II})$ complexes under investigation. The theoretical considerations herein predict extremely strong magnetic anisotropy of the titled compounds with $C_{3}$ easy axis of magnetization.

Acknowledgment. Financial support of U.S. Civilian Research and Development Foundation (CRDF), and Moldovan Research and Development Association (MRDA) (Award MP23022), National Science Foundation Nanoscale Science and Engineering NIRT Grant DMR-0103455, and Supreme Council on Science and Technological Development of Moldova (Grant 111) is highly appreciated.

\section{Appendix I}

The determinant wave functions of a $\mathrm{d}^{5}$ ion for the selected terms, $M_{\mathrm{S}}=S$ (symbol $M_{\mathrm{S}}$ is omitted), follow; only one basis function from each degenerate term is given; cubic basis related to the tetragonal axes is used.

$$
\begin{aligned}
& \left|\mathrm{t}_{2}^{5}, \mathrm{~T}_{2} \xi \frac{1}{2}\right\rangle=|\xi \eta \bar{\eta} \zeta \bar{\zeta}| \\
& \left|\mathrm{t}_{2}^{4}\left({ }^{1} \mathrm{~T}_{2}\right) \mathrm{e}\left({ }^{2} \mathrm{E}\right)^{2} \mathrm{~T}_{2} \xi \frac{1}{2}\right|=-\frac{1}{2 \sqrt{2}}|\xi \bar{\xi} \bar{\eta} \varsigma u|+\frac{1}{2 \sqrt{2}}|\xi \bar{\xi} \eta \bar{\zeta} u|+ \\
& \frac{\sqrt{3}}{2 \sqrt{2}}|\xi \bar{\xi} \bar{\eta} \varsigma v|-\frac{\sqrt{3}}{2 \sqrt{2}}|\xi \bar{\xi} \eta \bar{\zeta} v|
\end{aligned}
$$

$\left|\mathrm{t}_{2}^{4}\left({ }^{3} \mathrm{~T}_{1}\right) \mathrm{e}\left({ }^{2} \mathrm{E}\right)^{2} \mathrm{~T}_{2} \xi \frac{1}{2}\right\rangle=-\frac{1}{\sqrt{2}}|\xi \bar{\xi} \eta \zeta \bar{u}|+\frac{1}{2 \sqrt{2}}|\xi \bar{\xi} \bar{\eta} \varsigma u|+$

$$
\frac{1}{2 \sqrt{2}}|\xi \bar{\xi} \eta \bar{\zeta} u|-\frac{1}{\sqrt{6}}|\xi \bar{\xi} \eta \varsigma \bar{v}|+\frac{1}{2 \sqrt{6}}|\xi \bar{\xi} \bar{\eta} \varsigma v|+\frac{1}{2 \sqrt{6}}|\xi \bar{\xi} \eta \bar{\zeta} v|
$$$$
\left|\mathrm{t}_{2}^{4}\left({ }^{3} \mathrm{~T}_{1}\right) \mathrm{e}\left({ }^{2} \mathrm{E}\right)^{2} \mathrm{~T}_{1} \alpha \frac{1}{2}\right\rangle=\frac{1}{\sqrt{6}}|\xi \bar{\xi} \eta \varsigma \bar{u}|-\frac{1}{2 \sqrt{6}}|\xi \bar{\xi} \eta \bar{\zeta} u|-
$$$$
\frac{1}{2 \sqrt{6}}|\xi \bar{\xi} \bar{\eta} \varsigma u|-\frac{1}{\sqrt{2}}|\xi \bar{\xi} \eta \varsigma \bar{v}|+\frac{1}{2 \sqrt{2}}|\xi \bar{\xi} \eta \bar{\zeta} v|+\frac{1}{2 \sqrt{2}}|\xi \bar{\xi} \bar{\eta} \varsigma v|
$$$$
\left|\mathrm{t}_{2}^{4}\left({ }^{1} \mathrm{~T}_{2}\right) \mathrm{e}\left({ }^{2} \mathrm{E}\right)^{2} \mathrm{~T}_{1} \alpha \frac{1}{2}\right\rangle=-\frac{\sqrt{3}}{2 \sqrt{2}}|\xi \bar{\xi} \bar{\eta} \varsigma u|+\frac{\sqrt{3}}{2 \sqrt{2}}|\xi \bar{\xi} \eta \bar{\zeta} u|-
$$$$
\frac{1}{2 \sqrt{2}}|\xi \bar{\xi} \bar{\eta} \varsigma v|+\frac{1}{2 \sqrt{2}}|\xi \bar{\xi} \eta \bar{\zeta} v|
$$

$$
\begin{aligned}
\left|\mathrm{t}_{2}^{4}\left({ }^{1} \mathrm{E}\right) \mathrm{e}\left({ }^{2} \mathrm{E}\right)^{2} \mathrm{E} u \frac{1}{2}\right\rangle= & \frac{1}{2 \sqrt{3}}|\eta \bar{\eta} \zeta \bar{\zeta} u|+\frac{1}{2 \sqrt{3}}|\xi \bar{\xi} \zeta \bar{\zeta} u|- \\
& \frac{1}{\sqrt{3}}|\xi \bar{\xi} \eta \bar{\eta} u|+\frac{1}{2}|\eta \bar{\eta} \varsigma \bar{\zeta} v|-\frac{1}{2}|\xi \bar{\xi} \zeta \bar{\zeta} v|
\end{aligned}
$$

$$
\begin{aligned}
&\left|\mathrm{t}_{2}^{4}\left({ }^{1} \mathrm{~A}_{1}\right) \mathrm{e}\left({ }^{2} \mathrm{E}\right)^{2} \mathrm{E} u \frac{1}{2}\right\rangle=\frac{1}{\sqrt{3}}(|\xi \bar{\xi} \eta \bar{\eta} u|+|\xi \bar{\xi} \varsigma \bar{\zeta} u|+|\eta \bar{\eta} \varsigma \bar{\zeta} u|) \\
&\left|\mathrm{t}_{2}^{4}\left({ }^{1} \mathrm{E}\right) \mathrm{e}\left({ }^{2} \mathrm{E}\right)^{2} \mathrm{~A}_{2} \frac{1}{2}\right\rangle=-\frac{1}{2 \sqrt{3}}|\eta \bar{\eta} \varsigma \bar{\zeta} v|-\frac{1}{2 \sqrt{3}}|\xi \bar{\xi} \zeta \bar{\zeta} v|+ \\
& \frac{1}{\sqrt{3}}|\xi \bar{\xi} \eta \bar{\eta} v|-\frac{1}{2}|\eta \bar{\eta} \zeta \bar{\zeta} u|+\frac{1}{2}|\xi \bar{\xi} \varsigma \bar{\zeta} u|
\end{aligned}
$$

$$
\begin{aligned}
& \left|\mathrm{t}_{2}^{4}\left({ }^{3} \mathrm{~T}_{1}\right) \mathrm{e}\left({ }^{2} \mathrm{E}\right)^{4} \mathrm{~T}_{1} \alpha \frac{3}{2}\right\rangle=\frac{1}{2}|\xi \bar{\xi} \eta \zeta u|-\frac{\sqrt{3}}{2}|\xi \bar{\xi} \eta \varsigma v| \\
& \left.\mid \mathrm{t}_{2}{ }^{4}{ }^{3} \mathrm{~T}_{1}\right) \mathrm{e}\left({ }^{2} \mathrm{E}\right)^{4} \mathrm{~T}_{2} \xi \frac{3}{2}\left|=-\frac{\sqrt{3}}{2}\right| \xi \bar{\xi} \eta \zeta u\left|-\frac{1}{2}\right| \xi \bar{\xi} \eta \zeta v \mid \\
& \left|\mathrm{t}_{2}^{3}\left({ }^{2} \mathrm{~T}_{1}\right) \mathrm{e}^{2}\left({ }^{3} \mathrm{~A}_{2}\right){ }^{4} \mathrm{~T}_{2} \xi \frac{3}{2}\right|=\frac{1}{\sqrt{2}}(|\xi \eta \bar{\eta} u v|-|\xi \varsigma \bar{\zeta} u v|) \\
& \left|\mathrm{t}_{2}^{3}\left({ }^{4} \mathrm{~A}_{2}\right) \mathrm{e}^{2}\left({ }^{3} \mathrm{~A}_{2}\right){ }^{6} \mathrm{~A}_{1} \frac{5}{2}\right|=|\xi \eta \zeta u v|
\end{aligned}
$$

\section{Appendix II}

Reduced matrix elements of the trigonal crystal field operator $\mathbf{V}_{\mathbf{T}_{2} x_{0}}$ are

$$
\begin{gathered}
\left\langle\mathrm{t}_{2}^{4}\left({ }^{3} \mathrm{~T}_{1}\right) \mathrm{e}\left({ }^{2} \mathrm{E}\right)^{4} \mathrm{~T}_{1} \| \mathbf{V}_{\mathrm{T}_{2}}|| \mathrm{t}_{2}^{4}\left({ }^{3} \mathrm{~T}_{1}\right) \mathrm{e}\left({ }^{2} \mathrm{E}\right){ }^{4} \mathrm{~T}_{1}\right\rangle=-\frac{1}{\sqrt{2}} v, \\
\left.\left\langle\mathrm{t}_{2}^{4}\left({ }^{3} \mathrm{~T}_{1}\right) \mathrm{e}\left({ }^{2} \mathrm{E}\right){ }^{4} \mathrm{~T}_{1} \| \mathbf{V}_{\mathrm{T}_{2}}|| \mathrm{t}_{2}^{4}{ }^{3}{ }^{3} \mathrm{~T}_{1}\right) \mathrm{e}\left({ }^{2} \mathrm{E}\right)^{4} \mathrm{~T}_{2}\right\rangle=\sqrt{\frac{3}{2}} v, \\
\left\langle\mathrm{t}_{2}^{4}\left({ }^{3} \mathrm{~T}_{1}\right) \mathrm{e}\left({ }^{2} \mathrm{E}\right)^{4} \mathrm{~T}_{1} \| \mathbf{V}_{\mathrm{T}_{2}}|| \mathrm{t}_{2}^{3}\left({ }^{2} \mathrm{~T}_{1}\right) \mathrm{e}^{2}\left({ }^{3} \mathrm{~A}_{2}\right){ }^{4} \mathrm{~T}_{2}\right\rangle=\frac{3}{\sqrt{2}} v^{\prime}, \\
\left.\left\langle\mathrm{t}_{2}^{4}{ }^{3} \mathrm{~T}_{1}\right) \mathrm{e}\left({ }^{2} \mathrm{E}\right)^{4} \mathrm{~T}_{2}|| \mathbf{V}_{\mathrm{T}_{2}}|| \mathrm{t}_{2}^{4}\left({ }^{3} \mathrm{~T}_{1}\right) \mathrm{e}\left({ }^{2} \mathrm{E}\right){ }^{4} \mathrm{~T}_{2}\right\rangle=-\frac{1}{\sqrt{2}} v,
\end{gathered}
$$$$
\left.\left\langle\mathrm{t}_{2}^{4}{ }^{3} \mathrm{~T}_{1}\right) \mathrm{e}\left({ }^{2} \mathrm{E}\right)^{4} \mathrm{~T}_{2}|| \mathbf{V}_{\mathbf{T}_{2}}|| \mathrm{t}_{2}^{3}\left({ }^{2} \mathrm{~T}_{1}\right) \mathrm{e}^{2}\left({ }^{3} \mathrm{~A}_{2}\right){ }^{4} \mathrm{~T}_{2}\right\rangle=\frac{\sqrt{6}}{2} v^{\prime},
$$$$
\left\langle\mathrm{t}_{2}^{5,}, \mathrm{~T}_{2}|| \mathbf{V}_{\mathbf{T}_{2}}|| \mathrm{t}_{2}^{5}, \mathrm{~T}_{2}\right\rangle=-\sqrt{2} v
$$$$
\left\langle\mathrm{t}_{2}^{5}, \mathrm{~T}_{2}\left\|\mathbf{V}_{\mathbf{T}_{2}}\right\| \mathrm{t}_{2}^{4}\left({ }^{3} \mathrm{~T}_{1}\right) \mathrm{e}\left({ }^{2} \mathrm{E}\right)^{2} \mathrm{~T}_{2}\right\rangle=-\frac{3 \sqrt{6}}{2} v^{\prime},
$$

$$
\left\langle\mathrm{t}_{2}^{5}, \mathrm{~T}_{2}|| \mathbf{V}_{\mathrm{T}_{2}}|| \mathrm{t}_{2}^{4}\left({ }^{1} \mathrm{~T}_{2}\right) \mathrm{e}\left({ }^{2} \mathrm{E}\right)^{2} \mathrm{~T}_{2}\right\rangle=-\frac{\sqrt{6}}{2} v^{\prime},
$$

$\left\langle\mathrm{t}_{2}^{4}\left({ }^{3} \mathrm{~T}_{1}\right) \mathrm{e}\left({ }^{2} \mathrm{E}\right)^{2} \mathrm{~T}_{2}|| \mathbf{V}_{\mathrm{T}_{2}}|| \mathrm{t}_{2}^{4}\left({ }^{3} \mathrm{~T}_{1}\right) \mathrm{e}\left({ }^{2} \mathrm{E}\right)^{2} \mathrm{~T}_{2}\right\rangle=-\frac{1}{\sqrt{2}} v$,

$$
\left\langle\mathrm{t}_{2}^{4}\left({ }^{1} \mathrm{~T}_{2}\right) \mathrm{e}\left({ }^{2} \mathrm{E}\right)^{2} \mathrm{~T}_{2}|| \mathbf{V}_{\mathrm{T}_{2}}|| \mathrm{t}_{2}^{4}\left({ }^{1} \mathrm{~T}_{2}\right) \mathrm{e}\left({ }^{2} \mathrm{E}\right)^{2} \mathrm{~T}_{2}\right\rangle=\frac{1}{\sqrt{2}} v,
$$

$\left\langle\mathrm{t}_{2}^{4}\left({ }^{3} \mathrm{~T}_{1}\right) \mathrm{e}\left({ }^{2} \mathrm{E}\right)^{2} \mathrm{~T}_{1}|| \mathbf{V}_{\mathrm{T}_{2}}|| \mathrm{t}_{2}^{4}\left({ }^{3} \mathrm{~T}_{1}\right) \mathrm{e}\left({ }^{2} \mathrm{E}\right)^{2} \mathrm{~T}_{1}\right\rangle=-\frac{1}{\sqrt{2}} v$,

$$
\left\langle\mathrm{t}_{2}^{4}\left({ }^{1} \mathrm{~T}_{2}\right) \mathrm{e}\left({ }^{2} \mathrm{E}\right)^{2} \mathrm{~T}_{1}|| \mathbf{V}_{\mathbf{T}_{2}}|| \mathrm{t}_{2}^{4}\left({ }^{1} \mathrm{~T}_{2}\right) \mathrm{e}\left({ }^{2} \mathrm{E}\right)^{2} \mathrm{~T}_{1}\right\rangle=\frac{1}{\sqrt{2}} v,
$$

$\left\langle\mathrm{t}_{2}^{4}\left({ }^{1} \mathrm{E}\right) \mathrm{e}\left({ }^{2} \mathrm{E}\right)^{2} \mathrm{~A}_{2}\left\|\mathbf{V}_{\mathrm{T}_{2}}\right\| \mathrm{t}_{2}^{4}\left({ }^{1} \mathrm{~T}_{2}\right) \mathrm{e}\left({ }^{2} \mathrm{E}\right)^{2} \mathrm{~T}_{1}\right\rangle=-\sqrt{\frac{2}{3}} v$,
$\left\langle\mathrm{t}_{2}^{4}\left({ }^{1} \mathrm{~A}_{1}\right) \mathrm{e}\left({ }^{2} \mathrm{E}\right)^{2} \mathrm{E}\left\|\mathbf{V}_{\mathrm{T}_{2}}\right\| \mathrm{t}_{2}^{4}\left({ }^{1} \mathrm{~T}_{2}\right) \mathrm{e}\left({ }^{2} \mathrm{E}\right)^{2} \mathrm{~T}_{1}\right\rangle=-\frac{2 \sqrt{2}}{\sqrt{3}} v$,

$\left\langle\mathrm{t}_{2}^{4}\left({ }^{1} \mathrm{E}\right) \mathrm{e}\left({ }^{2} \mathrm{E}\right)^{2} \mathrm{E}|| \mathbf{V}_{\mathrm{T}_{2}}|| \mathrm{t}_{2}^{4}\left({ }^{1} \mathrm{~T}_{2}\right) \mathrm{e}\left({ }^{2} \mathrm{E}\right)^{2} \mathrm{~T}_{1}\right\rangle=\sqrt{\frac{2}{3}} v$,

$$
\left\langle\mathrm{t}_{2}^{4}\left({ }^{1} \mathrm{~A}_{1}\right) \mathrm{e}\left({ }^{2} \mathrm{E}\right)^{2} \mathrm{E}|| \mathbf{V}_{\mathrm{T}_{2}}|| \mathrm{t}_{2}^{5}, \mathrm{~T}_{2}\right\rangle=\sqrt{2} v^{\prime},
$$

$\left\langle\mathrm{t}_{2}^{4}\left({ }^{1} \mathrm{E}\right) \mathrm{e}\left({ }^{2} \mathrm{E}\right)^{2} \mathrm{E} \| \mathbf{V}_{\mathbf{T}_{2}}|| \mathrm{t}_{2}^{5},{ }^{2} \mathrm{~T}_{2}\right\rangle=-\sqrt{2} v^{\prime}$,

$$
\left\langle\mathrm{t}_{2}^{4}\left({ }^{1} \mathrm{~A}_{1}\right) \mathrm{e}\left({ }^{2} \mathrm{E}\right)^{2} \mathrm{E}|| \mathbf{V}_{\mathbf{T}_{2}}|| \mathrm{t}_{2}^{4}\left({ }^{1} \mathrm{~T}_{2}\right) \mathrm{e}\left({ }^{2} \mathrm{E}\right)^{2} \mathrm{~T}_{2}\right\rangle=-\frac{2 \sqrt{2}}{\sqrt{3}} v,
$$

$\left\langle\mathrm{t}_{2}^{4}\left({ }^{1} \mathrm{E}\right) \mathrm{e}\left({ }^{2} \mathrm{E}\right)^{2} \mathrm{E} \| \mathbf{V}_{\mathrm{T}_{2}}|| \mathrm{t}_{2}^{4}\left({ }^{1} \mathrm{~T}_{2}\right) \mathrm{e}\left({ }^{2} \mathrm{E}\right)^{2} \mathrm{~T}_{2}\right\rangle=-\sqrt{\frac{2}{3}} v$,

$$
\left\langle\mathrm{t}_{2}^{5}, \mathrm{~T}_{2}|| \mathbf{V}_{\mathbf{T}_{2}}|| \mathrm{t}_{2}^{4}\left({ }^{3} \mathrm{~T}_{1}\right) \mathrm{e}\left({ }^{2} \mathrm{E}\right)^{2} \mathrm{~T}_{1}\right\rangle=\frac{3}{\sqrt{2}} v^{\prime},
$$




$$
\begin{aligned}
\left\langle\mathrm{t}_{2}^{5},{ }^{2} \mathrm{~T}_{2}|| \mathbf{V}_{\mathbf{T}_{2}}|| \mathrm{t}_{2}^{4}\left({ }^{1} \mathrm{~T}_{2}\right) \mathrm{e}\left({ }^{2} \mathrm{E}\right)^{2} \mathrm{~T}_{1}\right\rangle=-\frac{3}{\sqrt{2}} v^{\prime}, \\
\left\langle\mathrm{t}_{2}^{4}\left({ }^{3} \mathrm{~T}_{1}\right) \mathrm{e}\left({ }^{2} \mathrm{E}\right)^{2} \mathrm{~T}_{2}|| \mathbf{V}_{\mathrm{T}_{2}}|| \mathrm{t}_{2}^{4}\left({ }^{3} \mathrm{~T}_{1}\right) \mathrm{e}\left({ }^{2} \mathrm{E}\right)^{2} \mathrm{~T}_{1}\right\rangle=\sqrt{\frac{3}{2}} v,
\end{aligned}
$$$$
\left\langle\mathrm{t}_{2}^{4}\left({ }^{1} \mathrm{~T}_{2}\right) \mathrm{e}\left({ }^{2} \mathrm{E}\right)^{2} \mathrm{~T}_{2}|| \mathbf{V}_{\mathbf{T}_{2}}|| \mathrm{t}_{2}^{4}\left({ }^{1} \mathrm{~T}_{2}\right) \mathrm{e}\left({ }^{2} \mathrm{E}\right)^{2} \mathrm{~T}_{1}\right\rangle=-\sqrt{\frac{3}{2}} v
$$

\section{Appendix III}

Reduced matrix elements of the orbital angular momentum operator within the adopted basis set are

$$
\begin{aligned}
& \left\langle\mathrm{t}_{2}^{4}\left({ }^{3} \mathrm{~T}_{1}\right) \mathrm{e}\left({ }^{2} \mathrm{E}\right)^{4} \mathrm{~T}_{1}|| \mathbf{L}_{\mathbf{T}_{1}}|| \mathrm{t}_{2}^{4}\left({ }^{3} \mathrm{~T}_{1}\right) \mathrm{e}\left({ }^{2} \mathrm{E}\right)^{4} \mathrm{~T}_{1}\right\rangle=\sqrt{\frac{3}{2}} i, \\
& \left\langle\mathrm{t}_{2}^{4}\left({ }^{3} \mathrm{~T}_{1}\right) \mathrm{e}\left({ }^{2} \mathrm{E}\right)^{4} \mathrm{~T}_{1}|| \mathbf{L}_{\mathbf{T}_{1}}|| \mathrm{t}_{2}^{4}\left({ }^{3} \mathrm{~T}_{1}\right) \mathrm{e}\left({ }^{2} \mathrm{E}\right)^{4} \mathrm{~T}_{2}\right\rangle=\frac{3}{\sqrt{2}} i,
\end{aligned}
$$

$\left\langle\mathrm{t}_{2}^{4}\left({ }^{3} \mathrm{~T}_{1}\right) \mathrm{e}\left({ }^{2} \mathrm{E}\right)^{4} \mathrm{~T}_{1}|| \mathbf{L}_{\mathrm{T}_{1}}|| \mathrm{t}_{2}^{3}\left({ }^{2} \mathrm{~T}_{1}\right) \mathrm{e}^{2}\left({ }^{3} \mathrm{~A}_{2}\right)^{4} \mathrm{~T}_{2}\right\rangle=\sqrt{3} i$,

$$
\left\langle\mathrm{t}_{2}^{4}\left({ }^{3} \mathrm{~T}_{1}\right) \mathrm{e}\left({ }^{2} \mathrm{E}\right)^{4} \mathrm{~T}_{2}|| \mathbf{L}_{\mathrm{T}_{1}}|| \mathrm{t}_{2}^{4}\left({ }^{3} \mathrm{~T}_{1}\right) \mathrm{e}\left({ }^{2} \mathrm{E}\right)^{4} \mathrm{~T}_{2}\right\rangle=-\sqrt{\frac{3}{2}} i,
$$

$\left\langle\mathrm{t}_{2}^{4}\left({ }^{3} \mathrm{~T}_{1}\right) \mathrm{e}\left({ }^{2} \mathrm{E}\right)^{4} \mathrm{~T}_{2}|| \mathbf{L}_{\mathbf{T}_{1}}|| t_{2}^{3}\left({ }^{2} \mathrm{~T}_{1}\right) \mathrm{e}^{2}\left({ }^{3} \mathrm{~A}_{2}\right)^{4} \mathrm{~T}_{2}\right\rangle=-3 i$,

$$
\left\langle\mathrm{t}_{2}^{3}\left({ }^{2} \mathrm{~T}_{1}\right) \mathrm{e}^{2}\left({ }^{3} \mathrm{~A}_{2}\right){ }^{4} \mathrm{~T}_{2} \| \mathbf{L}_{\mathrm{T}_{1}}|| \mathrm{t}_{2}^{3}\left({ }^{2} \mathrm{~T}_{1}\right) \mathrm{e}^{2}\left({ }^{3} \mathrm{~A}_{2}\right)^{4} \mathrm{~T}_{2}\right\rangle=-\sqrt{6} i,
$$

$\left\langle\mathrm{t}_{2}^{5},{ }^{2} \mathrm{~T}_{2}|| \mathbf{L}_{\mathbf{T}_{1}}|| \mathrm{t}_{2}^{5}, \mathrm{~T}_{2}\right\rangle=\sqrt{6} i$,

$$
\left\langle\mathrm{t}_{2}^{5},{ }^{2} \mathrm{~T}_{2}|| \mathbf{L}_{\mathbf{T}_{1}}|| \mathrm{t}_{2}^{4}\left({ }^{3} \mathrm{~T}_{1}\right) \mathrm{e}\left({ }^{2} \mathrm{E}\right)^{2} \mathrm{~T}_{2}\right\rangle=3 i,
$$

$\left\langle\mathrm{t}_{2}^{5},{ }^{2} \mathrm{~T}_{2}|| \mathbf{L}_{\mathbf{T}_{1}}|| \mathrm{t}_{2}^{4}\left({ }^{1} \mathrm{~T}_{2}\right) \mathrm{e}\left({ }^{2} \mathrm{E}\right)^{2} \mathrm{~T}_{2}\right\rangle=-3 i$,

$$
\left\langle\mathrm{t}_{2}^{4}\left({ }^{3} \mathrm{~T}_{1}\right) \mathrm{e}\left({ }^{2} \mathrm{E}\right)^{2} \mathrm{~T}_{2}|| \mathbf{L}_{\mathbf{T}_{1}}|| \mathrm{t}_{2}^{4}\left({ }^{3} \mathrm{~T}_{1}\right) \mathrm{e}\left({ }^{2} \mathrm{E}\right)^{2} \mathrm{~T}_{2}\right\rangle=-\sqrt{\frac{3}{2}} i,
$$

$\left\langle\mathrm{t}_{2}^{4}\left({ }^{1} \mathrm{~T}_{2}\right) \mathrm{e}\left({ }^{2} \mathrm{E}\right)^{2} \mathrm{~T}_{2}|| \mathbf{L}_{\mathrm{T}_{1}}|| \mathrm{t}_{2}^{4}\left({ }^{1} \mathrm{~T}_{2}\right) \mathrm{e}\left({ }^{2} \mathrm{E}\right)^{2} \mathrm{~T}_{2}\right\rangle=\sqrt{\frac{3}{2}} i$

$$
\left\langle\mathrm{t}_{2}^{4}\left({ }^{3} \mathrm{~T}_{1}\right) \mathrm{e}\left({ }^{2} \mathrm{E}\right)^{2} \mathrm{~T}_{1}|| \mathbf{L}_{\mathbf{T}_{1}}|| \mathrm{t}_{2}^{4}\left({ }^{3} \mathrm{~T}_{1}\right) \mathrm{e}\left({ }^{2} \mathrm{E}\right)^{2} \mathrm{~T}_{1}\right\rangle=\sqrt{\frac{3}{2}} i,
$$

$$
\begin{aligned}
& \left\langle\mathrm{t}_{2}^{4}\left({ }^{1} \mathrm{~T}_{2}\right) \mathrm{e}\left({ }^{2} \mathrm{E}\right)^{2} \mathrm{~T}_{1}|| \mathbf{L}_{\mathbf{T}_{1}}|| \mathrm{t}_{2}^{4}\left({ }^{1} \mathrm{~T}_{2}\right) \mathrm{e}\left({ }^{2} \mathrm{E}\right)^{2} \mathrm{~T}_{1}\right\rangle=-\sqrt{\frac{3}{2}} i, \\
& \left\langle\mathrm{t}_{2}^{4}\left({ }^{1} \mathrm{E}\right) \mathrm{e}\left({ }^{2} \mathrm{E}\right)^{2} \mathrm{E}|| \mathbf{L}_{\mathbf{T}_{1}}|| \mathrm{t}_{2}^{4}\left({ }^{1} \mathrm{~T}_{2}\right) \mathrm{e}\left({ }^{2} \mathrm{E}\right)^{2} \mathrm{~T}_{2}\right\rangle=\sqrt{6} i,
\end{aligned}
$$

$\left\langle\mathrm{t}_{2}^{4}\left({ }^{1} \mathrm{~A}_{1}\right) \mathrm{e}\left({ }^{2} \mathrm{E}\right)^{2} \mathrm{E}|| \mathbf{L}_{\mathbf{T}_{1}}|| t_{2}^{5},{ }^{2} \mathrm{~T}_{2}\right\rangle=-2 i$,

$$
\left\langle\mathrm{t}_{2}^{4}\left({ }^{1} \mathrm{E}\right) \mathrm{e}\left({ }^{2} \mathrm{E}\right)^{2} \mathrm{E}|| \mathbf{L}_{\mathbf{T}_{1}}|| t_{2}^{5}, \mathrm{~T}_{2}\right\rangle=-2 i,
$$

$\left\langle\mathrm{t}_{2}^{4}\left({ }^{1} \mathrm{E}\right) \mathrm{e}\left({ }^{2} \mathrm{E}\right)^{2} \mathrm{E}|| \mathbf{L}_{\mathrm{T}_{1}}|| \mathrm{t}_{2}^{4}\left({ }^{1} \mathrm{~T}_{2}\right) \mathrm{e}\left({ }^{2} \mathrm{E}\right)^{2} \mathrm{~T}_{1}\right\rangle=-\sqrt{6} i$,

$$
\left\langle\mathrm{t}_{2}^{4}\left({ }^{1} \mathrm{E}\right) \mathrm{e}\left({ }^{2} \mathrm{E}\right)^{2} \mathrm{~A}_{2}|| \mathbf{L}_{\mathrm{T}_{1}}|| \mathrm{t}_{2}^{4}\left({ }^{1} \mathrm{~T}_{2}\right) \mathrm{e}\left({ }^{2} \mathrm{E}\right)^{2} \mathrm{~T}_{2}\right\rangle=\sqrt{6} i,
$$

$$
\begin{gathered}
\left\langle\mathrm{t}_{2}^{5}, \mathrm{~T}_{2}|| \mathbf{L}_{\mathrm{T}_{1}} \| \mathrm{t}_{2}^{4}\left({ }^{3} \mathrm{~T}_{1}\right) \mathrm{e}\left({ }^{2} \mathrm{E}\right)^{2} \mathrm{~T}_{1}\right\rangle=3 \sqrt{3} i, \\
\left\langle\mathrm{t}_{2}^{5}, \mathrm{~T}_{2}|| \mathbf{L}_{\mathrm{T}_{1}} \| \mathrm{t}_{2}^{4}\left({ }^{1} \mathrm{~T}_{2}\right) \mathrm{e}\left({ }^{2} \mathrm{E}\right)^{2} \mathrm{~T}_{1}\right\rangle=\sqrt{3} i, \\
\left\langle\mathrm{t}_{2}^{4}\left({ }^{3} \mathrm{~T}_{1}\right) \mathrm{e}\left({ }^{2} \mathrm{E}\right)^{2} \mathrm{~T}_{2} \| \mathbf{L}_{\mathrm{T}_{1}}|| \mathrm{t}_{2}^{4}\left({ }^{3} \mathrm{~T}_{1}\right) \mathrm{e}\left({ }^{2} \mathrm{E}\right)^{2} \mathrm{~T}_{1}\right\rangle=\frac{3}{\sqrt{2}} i, \\
\left\langle\mathrm{t}_{2}^{4}\left({ }^{1} \mathrm{~T}_{2}\right) \mathrm{e}\left({ }^{2} \mathrm{E}\right)^{2} \mathrm{~T}_{2}|| \mathbf{L}_{\mathrm{T}_{1}}|| \mathrm{t}_{2}^{4}\left({ }^{1} \mathrm{~T}_{2}\right) \mathrm{e}\left({ }^{2} \mathrm{E}\right)^{2} \mathrm{~T}_{1}\right\rangle=-\frac{3}{\sqrt{2}} i,
\end{gathered}
$$$$
\left\langle\mathrm{t}_{2}^{4}\left({ }^{1} \mathrm{E}\right) \mathrm{e}\left({ }^{2} \mathrm{E}\right)^{2} \mathrm{~A}_{2} \| \mathbf{L}_{\mathbf{T}_{1}}|| t_{2}^{5,}, \mathrm{~T}_{2}\right\rangle=2 i
$$

\section{References and Notes}

(1) Sugano, S.; Tanabe, Y.; Kamimura, H. Multiplets of Transition Metal Ions in Crystals; Academic Press: London, U.K., 1970.

(2) Griffith, J. S. The Theory of Transition-Metal Ions; University Press: Cambridge, U.K., 1961.

(3) Kahn, O. Molecular Magnetism; VCH Publishers: New York, 1993.

(4) Bencini, A.; Gatteschi, D. Electron Paramagnetic Resonance of Exchange Coupled Systems; Springer-Verlag: Berlin, 1990.

(5) Lever, A. B. Inorganic Electronic Spectroscopy; Elsevier: Amsterdam, 1984.

(6) Chakravarty, A. C. Introduction to the Magnetic Properties of Solids; Wiley: New York, 1980.

(7) Van Vleck, J. H. The Theory of Electric and Magnetic Susceptibilities; Clarendon Press: Oxford, U.K., 1932.

(8) Liehr, A. D. J. Phys. Chem. 1960, 64, 43.

(9) Moffit, W. E.; Goodman, G. L.; Freed, M.; Weinstock, B. Mol. Phys. 1959, 2, 109.

(10) Caneschi, A.; Gattteschi, D.; Sessoli, R.; Novak, M. A. Nature 1993, 365,141 .

(11) Barbara, B.; Gunther, L. Phys. World 1999, 12, 35.

(12) Uzelmeier, C. E.; Bartley, S. L.; Fourmigue, M.; Rogers, R.; Grandinetti, G.; Dunbar, K. R. Inorg. Chem. 1998, 37, 6706.

(13) (a) Schelter, E. J.; Bera, J. K.; Bacsa, Galán-Mascarós, J. R.; Dunbar, K. R. Inorg. Chem. 2003, 42, 4256-4258. (b) Bartley, S. L.; Bazile, M. J.; Clérac, R.; Zhao, H.; Ouyang, X.; Dunbar, K. R. Dalton Trans. 2003, 2937-2944.

(14) Thornley, J. H. M. J. Phys. C 1968, 1, 1024.

(15) Pryce, M. H. L.; Runciman, W. A. Discuss. Faraday Soc. 1958, $26,34$.

(16) Figgis, B. N. Introduction to Ligand Fields; Interscience: New York, 1966.

(17) Hall, M. B. J. Am. Chem. Soc. 1975, 97, 2057.

(18) Varshalovich, D. A.; Moskalev, A. N.; Khersonskii, V. K. Quantum Theory of Angular Momentum; World Scientific: Singapore, 1988.

(19) Dunbar, K.; Schelter, E.; Tsukerblat, B. S.; Palii, A. V.; Ostrovsky, S. M.; Mirovitskii, V. Yu.; Klokishner, S. I. Adv. Quantum Chem., in press.

(20) Dunbar, K.; Schelter, E.; Tsukerblat, B. S.; Ostrovsky, S. M.; Mirovitskii, V. Yu.; Palii, A. V. Polyhedron 2003, 14-17, 25452556.

(21) Pope, M. T. Heteropoly and Polyoxometalates; Springer: Berlin, 1983.

(22) Allen, G. C.; Al-Mobarak, R.; El-Sharkawy, G. A. M.; Warren, K. D. Inorg. Chem. 1972, 11, 787. 